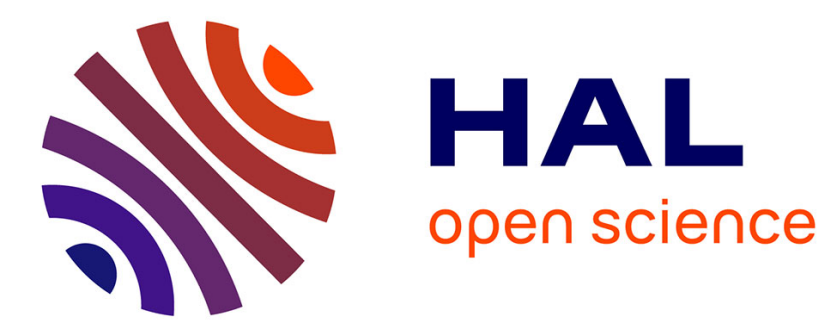

\title{
Thermodynamic stability of solid and fluid phases in the Si3B3N7 system
}

\author{
Alexander Hannemann, Christian Schoen, Martin Jansen
}

\section{To cite this version:}

Alexander Hannemann, Christian Schoen, Martin Jansen. Thermodynamic stability of solid and fluid phases in the Si3B3N7 system. Philosophical Magazine, 2009, 88 (07), pp.1037-1057. 10.1080/14786430802047084 . hal-00513885

\section{HAL Id: hal-00513885 \\ https://hal.science/hal-00513885}

Submitted on 1 Sep 2010

HAL is a multi-disciplinary open access archive for the deposit and dissemination of scientific research documents, whether they are published or not. The documents may come from teaching and research institutions in France or abroad, or from public or private research centers.
L'archive ouverte pluridisciplinaire HAL, est destinée au dépôt et à la diffusion de documents scientifiques de niveau recherche, publiés ou non, émanant des établissements d'enseignement et de recherche français ou étrangers, des laboratoires publics ou privés. 


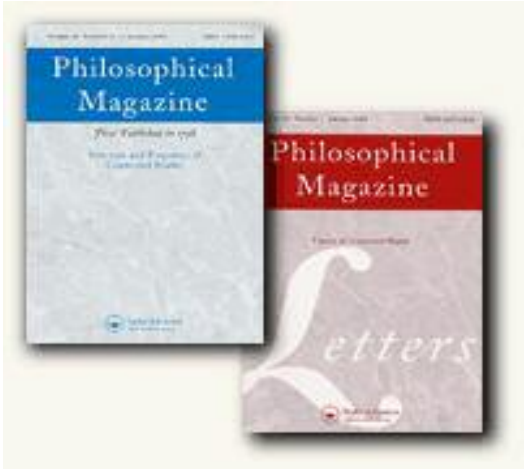

Thermodynamic stability of solid and fluid phases in the $\mathrm{Si}_{3} \mathrm{~B}_{3} \mathrm{~N}_{7}$ system

\begin{tabular}{|c|c|}
\hline Journal: & Philosophical Magazine \& Philosophical Magazine Letters \\
\hline Manuscript ID: & TPHM-07-Sep-0261 \\
\hline Journal Selection: & Philosophical Magazine \\
\hline $\begin{array}{r}\text { Date Submitted by the } \\
\text { Author: }\end{array}$ & 25-Sep-2007 \\
\hline Complete List of Authors: & $\begin{array}{l}\text { Hannemann, Alexander; FIZ Karlsruhe } \\
\text { Schoen, Christian; Max-Planck Institute for Solid State Research, } \\
\text { Jansen } \\
\text { Jansen, Martin; Max-Planck-Institute for Solid State Research, } \\
\text { Jansen }\end{array}$ \\
\hline Keywords: & $\begin{array}{l}\text { amorphous materials, computer simulation, glass ceramics, high- } \\
\text { temperature materials, Monte-Carlo, phase diagrams, phase } \\
\text { stability }\end{array}$ \\
\hline Keywords (user supplied): & silicon boron nitride \\
\hline \multicolumn{2}{|c|}{$\begin{array}{l}\text { Note: The following files were submitted by the author for peer review, but cannot be converted } \\
\text { to PDF. You must view these files (e.g. movies) online. }\end{array}$} \\
\hline $\begin{array}{l}\text { captionsthermodyn.tex } \\
\text { discussionthermodyn.tex } \\
\text { introthermodyn.tex } \\
\text { methodthermodyn.tex } \\
\text { paperthermodyn.tex } \\
\text { resultsthermodyn.tex } \\
\text { paperthermodyn.bbl }\end{array}$ & \\
\hline
\end{tabular}




\section{S. ScholarONE"}




\title{
Thermodynamic stability of solid and fluid phases in the $\mathrm{Si}_{3} \mathrm{~B}_{3} \mathrm{~N}_{7}$ system
}

\author{
A.Hannemann ${ }^{a}$, J.C. Schön, M. Jansen \\ Max-Planck-Institut für Festkörperforschung \\ Heisenbergstr. 1, D-70569 Stuttgart, Germany. \\ ${ }^{a}$ new address: FIZ Karlsruhe, Hermann-von-Helmholtz-Platz 1, \\ 76344 Eggenstein-Leopoldshafen
}

September 25, 2007

\begin{abstract}
We investigate the thermodynamic properties of the ceramic material $\mathrm{Si}_{3} \mathrm{~B}_{3} \mathrm{~N}_{7}$ which has so far only been synthesized as an amorphous compound. Using Monte Carlo simulations, we investigate the stability of both solid and fluid phases of $\mathrm{Si}_{3} \mathrm{~B}_{3} \mathrm{~N}_{7}$, in order to gain insights into the proper synthetic conditions needed to generate the stable amorphous and crystalline phases of this compound. We study the ternary liquid-gas region of the phase diagram at temperatures above the theoretical glass transition in this system, and construct an approximate "metastable" phase-diagram of $\mathrm{Si}_{3} \mathrm{~B}_{3} \mathrm{~N}_{7}$. In addition we study the stability of the amorphous and crystalline phases in the solid state against the decomposition into the binary phases h-BN and $\beta-\mathrm{Si}_{3} \mathrm{~N}_{4}$ as function of the size of the crystallites involved, and the stability of the melt against evolution of nitrogen as function of nitrogen pressure.
\end{abstract}

Pacs.No.: 81.30.Dz, 05.70.-a, 81.05.Je 


\section{Introduction}

One of the most fascinating new classes of high-technology materials are the amorphous nitridic ceramics of the composition $a-\mathrm{Si}_{x} \mathrm{~B}_{y} \mathrm{~N}_{1} \mathrm{C}_{z}[1,2,3,4,5,6,7,8,9,10,11]$ such as a- $\mathrm{Si}_{3} \mathrm{~B}_{3} \mathrm{~N}_{7}$ and a-SiBN ${ }_{3} \mathrm{C}$. These compounds are synthesized via the sol-gel route and exhibit a very high stability against crystallization up to $1900 \mathrm{~K}$ and $2100 \mathrm{~K}$ for a-Si $\mathrm{B}_{3} \mathrm{~B}_{3} \mathrm{~N}_{7}$ and a-SiBN ${ }_{3} \mathrm{C}$, respectively. Furthermore, e.g. a- $\mathrm{SiBN}_{3} \mathrm{C}$ is stable against oxidation up to $1700 \mathrm{~K}$, and exhibits a high bulk modulus $B=200-300 \mathrm{GPa}$, while at the same time the density of the materials is very low, e.g. $\rho \approx 1.9 \mathrm{~g} / \mathrm{cm}^{3}$ for a- $\mathrm{Si}_{3} \mathrm{~B}_{3} \mathrm{~N}_{7}$, compared to the weighted average of the binary endphases $\mathrm{Si}_{3} \mathrm{~N}_{4}$ and $\mathrm{BN}, \rho=2.8 \mathrm{~g} / \mathrm{cm}^{3}$.

Understanding the reason for this surprising stability is of great importance for the future design of such new materials. Regarding their structure, it has been found experimentally[11, $12,13]$ and within the context of simulations $[14,15]$ that the prototypical representative of this class of materials, a- $\mathrm{Si}_{3} \mathrm{~B}_{3} \mathrm{~N}_{7}$, is built up of random networks of $\mathrm{SiN}_{4}$-tetrahedra and trigonally planar coordinated $\mathrm{BN}_{3}$ - units. Here, the cation distribution is found to be homogeneous down to length scales of about $1 \mathrm{~nm}$, while below this value one finds a moderate tendency towards heterogeneity[16].

In previous work, we have generated models of a- $\mathrm{Si}_{3} \mathrm{~B}_{3} \mathrm{~N}_{7}$ via a detailed analysis of the actual sol-gel synthesis route[17, 18], which leads to a homogeneous amorphous compound of density $\rho \approx 1.8-2.0 \mathrm{~g} / \mathrm{cm}^{3}$ exhibiting voids of diameter between $1 / 2$ and 1 nanometer, and a slight amount of heterogeneity in the cation distribution on the sub-nanometer scale. We have investigated the stability of these void-containing structures[19] and have found that they should be kinetically stable up to ca. $1750 \mathrm{~K}$. Above this temperature, a very slow transformation into a denser amorphous phase without larger voids should take place - the same phase one would also obtain if one could synthesize a- $\mathrm{Si}_{3} \mathrm{~B}_{3} \mathrm{~N}_{7}$ via a glass transition from the melt[15] with a glass transition temperature somewhere in the range of $2000-2250 \mathrm{~K}$ according to our simulations[20]. Up to now, however, the melt phase of $\mathrm{Si}_{3} \mathrm{~B}_{3} \mathrm{~N}_{7}$ has not yet been accessible experimentally, most likely due to the evolution of $\mathrm{N}_{2}$ from the melt. Similarly, it has not been possible to generate an amorphous phase of 
$\mathrm{Si}_{3} \mathrm{~B}_{3} \mathrm{~N}_{7}$ by sintering of $1 / 2$-micron size particles of h-BN and $\beta$ - $\mathrm{Si}_{3} \mathrm{~N}_{4}$. [6] Both of these routes are of great interest, in principle, since they might serve both as an alternative to the conventional sol-gel approach and yield new amorphous phases of a- $\mathrm{Si}_{3} \mathrm{~B}_{3} \mathrm{~N}_{7}$ with different, possibly even better, physical properties.[15]

In order to gain further insight into the kinetic and thermodynamic stabilities of the ternary system $\mathrm{Si}_{3} \mathrm{~B}_{3} \mathrm{~N}_{7}$, we first investigate in this work the (metastable) phase diagram of $\mathrm{Si}_{3} \mathrm{~B}_{3} \mathrm{~N}_{7}$ restricted to this composition. One focus is on the (high-temperature) liquid-gas region above the glass transition temperature of the amorphous state and the melting temperature of a hypothetical crystalline modification of $\mathrm{Si}_{3} \mathrm{~B}_{3} \mathrm{~N}_{7}$. After a short description of the simulation method, we analyze the computed pressure data and the cluster size distributions as function of temperature and volume, and construct the phase diagram of the ternary $\mathrm{Si}_{3} \mathrm{~B}_{3} \mathrm{~N}_{7}$-fluid.

Of course, in the solid state region, one needs to take into account that both the amorphous and the hypothetical crystalline phase might decompose into the two binary boundary phases, h-BN and $\beta-\mathrm{Si}_{3} \mathrm{~N}_{4}$. Thus, we also study the stability of the ternary phases against the decomposition into h-BN and $\beta-\mathrm{Si}_{3} \mathrm{~N}_{4}$ as function of the size of the crystallites involved.

\section{Method}

\subsection{General procedure for phase diagram construction}

The route to constructing the phase diagram presented here consists of three steps:

1) First, we perform long simulations (mostly of the Monte Carlo random walk type) of $\mathrm{Si}_{3} \mathrm{~B}_{3} \mathrm{~N}_{7}$ for a large number of temperatures and volumes, covering the solid, liquid and gaseous state of the system. However, it is well-known that, for low temperatures, the time scales of the simulations are much too short for such a system to reach full equilibrium[21, 22]. Thus, we have performed two different sets of simulations that differed in the starting configuration, in order to be able to place the results into the proper 
context.

- In procedure 1 , the starting configurations for the simulations were generated by equilibration of a ternary melt, followed by a re-scaling to the volume under consideration. Thus, the initial atom arrangement could be denoted as a "stretched melt" configuration. For very large volumes, the subsequent process can be visualized as a condensation from the gas phase at low temperatures.

- Procedure 2 generated the starting configuration by placing the relaxed melt configuration unchanged at the center of the simulation cell. We called this arrangement a "free block" configuration, and the time evolution corresponds to the evaporation from the condensed phase.

2) For each such simulation, we analyze the trajectory of the system and check, whether the system is found in a condensed (solid/liquid) or gaseous state. As indicator for the condensed phase we use the existence / non-existence of a large cluster encompassing nearly all atoms in the simulation cell over the observed time window. Next, we distinguish between the solid and the liquid state by measuring the survival probability of the bonds in the condensed phase and the diffusivity. This is necessary, since it is rather difficult to use the average pair correlation function to detect the presence of a liquid vs. a solid but amorphous state ${ }^{1}$ (of course, the crystalline state would be easily detectable, but for the relatively short simulation times accessible, the formation of a crystalline structure from a random starting configuration in a system containing hundreds of atoms is usually not feasible.).

3) By noting for each temperature-volume data point whether either a solid phase (plus no or only a small amount of gas), a liquid phase (plus no or only a small amount of gas), a gaseous phase (plus no or only a small amount of liquid), a co-existence of a gaseous and a liquid phase, or only a gaseous phase, is present, we can finally construct

\footnotetext{
${ }^{1}$ The average pair correlation function is essentially a one-time property of the system, but distinguishing between glass and liquid requires the measurement of two-time properties representing the response to some external action, such as the viscosity.
} 
an approximate phase diagram of the system. Since procedures 1 and 2 can yield different phase assignments due to the finite length of the simulations, we have employed simple estimates of the difference in the free energies between the results of the two procedures, in order to decide, which assignment is the more appropriate one for a given volume and temperature.

We have chosen this rather unconventional approach instead of employing some more standard and/or refined methods used to estimate the free energies of a phase (see e.g. [23]) or to determine the thermodynamically stable phase by multi-histogram methods (see e.g. [24]), because of the computational cost involved in performing the necessary very long and rather complicated simulations for a large number of strongly interacting atoms in a partially covalent, partially ionic ternary system such as $\mathrm{Si}_{3} \mathrm{~B}_{3} \mathrm{~N}_{7}$. The complexity of this task is underlined by e.g. the fact that so far none of the many experiments trying to synthesize a crystalline modification of $\mathrm{Si}_{3} \mathrm{~B}_{3} \mathrm{~N}_{7}$ have succeeded - instead either the starting materials such as the binary end-phases or the ternary amorphous phase have been recovered, or there has been a complete decomposition of the starting materials.

\section{$2.2 \quad$ Model}

The model of $\mathrm{Si}_{3} \mathrm{~B}_{3} \mathrm{~N}_{7}$ we employed for the thermodynamical computations consisted of 162 Si-atoms, 162 B-atoms and $378 \mathrm{~N}$-atoms $\left(N_{\text {atom }}=702\right)$, respectively, in a periodically repeated cubic box. As an interaction potential, we employed a medium range two-body potential from the literature[25]. The parameters in the potential had been fitted to ab initio calculations for molecules containing Si-N and B-N bonds, for the binary crystalline compounds $\mathrm{BN}$ and $\mathrm{Si}_{3} \mathrm{~N}_{4}$, and for hypothetical crystalline ternary compounds $\mathrm{Si}_{3} \mathrm{~B}_{3} \mathrm{~N}_{7}$. The potential had been shown to satisfactorily reproduce the structure and vibrational properties of the binary compounds $\mathrm{Si}_{3} \mathrm{~N}_{4}$ and BN. ${ }^{2}$

The simulations were performed at fixed temperature and volume, with a Monte-Carlo

\footnotetext{
${ }^{2}$ Since the specific form of the potential is rather complex, we refer to the literature[25] for further details.
} 
algorithm using the Metropolis acceptance criterion. The moveclass consisted of single atom moves, adapted in size to achieve an acceptance rate of $\approx 50 \%$. One Monte-Carlo cycle (MCC) corresponded to $N_{\text {atom }}=702$ individual attempted moves. ${ }^{3}$

The temperatures investigated ranged from $250 \mathrm{~K}$ to $7000 \mathrm{~K}$, and the volumes (of the periodically repeated simulation cell) ranged from $5000 \AA^{3}$ to $2.5 \cdot 10^{6} \AA^{3}$, corresponding to number densities $\rho_{N}$ from 0.12 atoms $/ \AA^{3}$ to $10^{-5}$ atoms $/ \AA^{3}$. For each temperature and volume we performed runs of length $10^{6} \mathrm{MCC}$, resulting in about 600 data points for each of the two procedures described below. Since we had found that the system exhibits aging effects[26, 20], we have only used the last $2 \cdot 10^{5} \mathrm{MCC}$ (corresponding to a time window of about $100 \mathrm{ps}$ ) after a waiting time of $t_{w}=8 \cdot 10^{5} \mathrm{MCC}$, for analysis purposes in order to allow the system to reach at least quasi-equilibrium. Furthermore the runs were repeated 8 times at each temperature in order to improve the statistics of our simulations.

As mentioned above, we have performed two different sets of simulations that differed in the starting configuration, in order to be able to place the results into the proper context: the 'stretched melt' configuration (procedure 1) and the 'free block' configuration (procedure 2). In procedure 1, the starting configurations for the simulations were generated by equilibration of a ternary melt of $\mathrm{Si}_{3} \mathrm{~B}_{3} \mathrm{~N}_{7}[14]$ at $T=2000 \mathrm{~K}$ in a simulation cell of volume $19.1 \times 19.1 \times 19.1 \AA^{3} \approx 7000 \AA^{3}$, followed by a re-scaling to the volume under consideration. In procedure 2 , the same 'melt' has been placed inside the simulation cell without any rescaling. Finally, instead of a dense melt or amorphous solid, we have also employed a hypothetical crystalline polymorph[27] as a starting configuration in procedure 1.

\footnotetext{
${ }^{3} \mathrm{In}$ addition, MD simulations in the melt (at $2500 \mathrm{~K}$ ) were performed that allowed us to tentatively calibrate the time scale of the MC-simulations via a comparison of the mean square displacements (at least in the high-temperature region). We found that $1 \mathrm{MCC}$ corresponded to approximately $0.5 \mathrm{fs}$.
} 


\subsection{Analysis procedures}

In order to distinguish between solid, liquid and gaseous phases several criteria were used, which were based on one-time properties such as the (potential) energy, pressure and cluster distributions, as well as on two-point properties like the diffusion coefficient and the bond-survival probabilities. To analyze the one-time properties, we registered the potential energy $E_{p o t}$, the pressure $p$, the distribution of connected clusters, and the distribution of connected void regions every 100 MCC. The pressure was evaluated using[28]

$$
p=\rho_{N} k_{B} T-\frac{1}{3 V} \sum_{i}^{N} \sum_{j>i}^{N} \vec{r}_{i j} \frac{\partial V_{i n t}\left(r_{i j}\right)}{\partial \vec{r}_{i j}},
$$

where $V_{\text {int }}\left(r_{i j}\right)$ is the interaction potential between atoms $i$ and $j$ separated by a distance $r_{i j}$

Clusters were identified using a topology-based criterion. We defined bonds between atom pairs Si-N and B-N, if the distance between cation and anion was below $2.0 \AA$. Next, we employed a depth-first search algorithm[29] on the bond graph to determine the graph's connected components, yielding the cluster size distribution at time $t, h\left(S_{c l} ; t\right)$ $\left(S_{c l}=1, \ldots, N_{\text {atom }}\right)$.

From $h\left(S_{c l} ; t\right)$, we extracted the average cluster size at a given time $S_{\text {mean }}(t)$, the size of the largest cluster present $S_{\max }(t)$, and the number of clusters at time $t, N_{c l}(t)$. Furthermore, the distributions were averaged over the observation time of $2 \cdot 10^{5} \mathrm{MCC}$ (after a waiting time of $t_{w}=8 \cdot 10^{5} \mathrm{MCC}$ ), yielding $\left\langle h\left(S_{c l}\right)\right\rangle_{t},\left\langle S_{\text {mean }}\right\rangle_{t},\left\langle S_{\text {max }}\right\rangle_{t}$, and $\left\langle N_{c l}\right\rangle_{t}$. From $\left\langle h\left(S_{c l}\right)\right\rangle_{t}$, one can derive the likelihood $\left\langle L\left(S_{c l}\right)\right\rangle_{t}$ of an atom to be found as part of a cluster of size $S_{c l}$,

$$
\left\langle L\left(S_{c l}\right)\right\rangle_{t}=\frac{S_{c l} \cdot\left\langle h\left(S_{c l}\right)\right\rangle_{t}}{N_{\text {atom }}}
$$

For the two-time properties, we calculated the mean square displacement $M S D\left(t_{o b s} ; T, V\right)$ and the bond survival probabilities $B S P^{(S i N)}\left(t_{w}, t_{o b s} ; T, V\right)$ and $B S P^{(B N)}\left(t_{w}, t_{o b s} ; T, V\right)$, i.e. the likelihood that a given Si-N or B-N bond, respectively, observed at time $t_{w}\left(=5 \cdot 10^{5}\right.$ MCC) still exists after a time $t_{o b s}\left(=5 \cdot 10^{5} \mathrm{MCC}\right)$ has elapsed. From the mean squared 
displacement $M S D\left(t_{o b s}\right)$, we calculated the diffusion coefficients

$$
D(T, V)=\lim _{t_{o b s} \rightarrow \infty} \frac{M S D\left(t_{o b s} ; T, V\right)}{6 t_{o b s}}
$$

Note that in the non-equilibrium regime, equation 3 only gives an estimate for the diffusion coefficient, since in that regime the linear relationship between $t_{o b s}$ and the $M S D\left(t_{o b s} ; V, T\right)$ does not hold in general[20]. But for the present purpose this estimate suffices.

Two steps were required to assign a simulated system, for a given set of thermodynamic parameters $(V, T)$, to the solid, liquid or gaseous state. First we needed to identify the condensed states (solid and liquid) and the gaseous states, and in a second step, we had to distinguish between solid and liquid states. In contrast to a system in the thermodynamic $\operatorname{limit}\left(N_{\text {atom }}, V \rightarrow \infty\right.$, with $\rho_{N}=N_{\text {atom }} / V=$ constant $)$, the simulations can never realize a condensed phase of infinite size, and furthermore surface (i.e. interface between condensed and gas phase) effects need to be taken into account. Thus, in order to gain a quantitative handle that allows us to assign a label "condensed"/"gaseous" to a point $(V, T)$ in the phase diagram, we have chosen $S_{c l}^{*}=50$ as a delimiter, after careful inspection of the cluster size distributions we have obtained. If the likelihood to be part of a cluster with size $S_{c l} \leq S_{c l}^{*}$

$$
f\left(S_{c l}, S_{c l}^{*}\right)=1 / N_{a t o m} \sum_{S_{c l} \leq S_{c l}^{*}} S_{c l} \cdot\left\langle h\left(S_{c l}\right)\right\rangle_{t}=\sum_{S_{c l} \leq S_{c l}^{*}}\left\langle L\left(S_{c l}\right)\right\rangle_{t},
$$

exceeds a predefined cutoff $f_{\text {cut }}(=0.9)$, the system as a whole is considered to be in the gaseous phase, while for $f\left(S_{c l}, S_{c l}^{*}\right)<1-f_{c u t}(=0.1)$, the system as a whole is assigned to the condensed state. For intermediate values, the system was considered to be in the two-phase region, where a condensed and a gaseous phase co-exist. Even though these assignments to a condensed or a gaseous state depend on the choices of $S^{*}$ and $f_{\text {cut }}$, we found no significant effect on the final assignments for a wide range of these parameters (see figure 5).

In the second step, we have used the bond-survival probabilities as a criterion for distinguishing between the solid and the liquid state of $\mathrm{Si}_{3} \mathrm{~B}_{3} \mathrm{~N}_{7}$. We note that the structure 
remains essentially unchanged, if most of the bonds are preserved for a given observation time $t_{o b s}$. Thus, we called the condensed system liquid, if the BSPs for a condensed phase had dropped below a value $1 / e .^{4}$ On the other hand, if the BSPs for the condensed phase were larger than 0.8, we identified the system as an (amorphous) solid. Note that the "BSP-criterion" alone could not be used to distinguish between the gaseous state and the solid state, since a system consisting of gas molecules only, will also show a high value of the bond-survival probabilities. Here, the diffusion coefficient could be used as a criterion.

\section{Results}

\subsection{Ternary liquid-gas region}

Using procedure 1 outlined in the previous section, we have calculated the pressure at each given volume and temperature. Fig. 1(a) shows the so-called compression coefficient[30] $Z=p V / N_{\text {atom }} k_{B} T$ as a function of number density $\rho_{N}=N_{\text {atom }} / V$, which would equal one for an ideal gas. For a given temperature, $Z$ rapidly increases to high positive values for high densities, crosses zero near $\rho_{N}=0.1$ atoms $/ \AA^{3}$ (close to the equilibrium value for the starting melt, corresponding for $\mathrm{Si}_{3} \mathrm{~B}_{3} \mathrm{~N}_{7}$ to a mass density of $\rho_{m}=2.7 \mathrm{~g} / \mathrm{cm}^{3}$ ), exhibits a negative minimum value around $\rho_{N}=0.08$ atoms $/ \AA^{3}$, and then slowly increases monotonically for $\rho_{N} \rightarrow 0$. Fig. 1(b) shows the pressure computed according to eq. 1 for high temperatures. The general shape resembles the one observed for e.g. a van der Waals gas, but we do find negative pressures for densities below $\rho_{N}=0.08$ atoms $/ \AA^{3}$.

For the second procedure, $Z$ remains positive and moves relatively close to 1 for very high temperatures. Similarly, we see from figure $1(\mathrm{~b})$ that for the most part $p(V, T)>0$, dropping below zero only for low temperatures at intermediate densities $\left(0.01<\rho_{N}<0.1\right.$ atoms $\left(\AA^{3}\right)$. It appears that for the free block model the major contribution to the pressure stems from the few atoms that have evaporated from the condensed phase and behave

\footnotetext{
${ }^{4}$ Note that for a system that relaxes according to a Debye-law, a value of the BSPs larger than 1/e would imply that the relaxation times $\tau$ exceeded the observation time $t_{o b s}$.
} 
nearly like an ideal gas. In contrast, in the first procedure the negative pressures are presumably due to the energy loss associated with the formation of the comparatively large numbers of clusters starting at intermediate densities.

\subsection{Cluster size distribution in the fluid phase}

One conclusion from the preceding subsection 3.1, would be that $\mathrm{Si}_{3} \mathrm{~B}_{3} \mathrm{~N}_{7}$ does not necessarily behave like a simple fluid. Thus, within the range of our simulations, the mean-field picture is only marginally appropriate for a quantitative description. This agrees with our earlier work[26, 20] studying the glass transition: Judging from the specific heat curves, there appears to occur a considerable release of configurational entropy at temperatures far above the actual glass transition at $T_{G} \approx 2000-2500 \mathrm{~K}$ that was computed based on the peak in the specific heat and the decrease of the diffusion constants.

The reason for this disparity lies in the fact that upon melting / softening of the crystalline / amorphous solid, we do not quickly reach a phase consisting of individual atoms or molecules, respectively. Instead, we observe a wide distribution of long-lived $\mathrm{Si} / \mathrm{B} / \mathrm{N}$ clusters in the fluid phase(s), up to relatively high temperatures.

Fig. 2(a) and fig. 2(b) show the time-average of the average size of the observed clusters $\left\langle S_{\text {mean }}\right\rangle_{t}$, and the average number of clusters $\left\langle N_{c l}\right\rangle_{t}$ present, respectively, as a function of $T$ and $V$, for both procedures. As expected, the mean cluster size decreases with increasing temperature and decreasing density, for both procedures, although we note the markedly higher values at low temperatures and low densities for procedure 2.

However, the mean cluster size is not necessarily a very significant measure of the cluster distribution. While at low densities and high temperatures we would expect that the cluster sizes cluster around the average value, this might not be true for the low density-low temperature region. Here, we might deal with a large piece of solid surrounded by a few atoms or very small clusters in the gas phase. Thus, we show in fig. 2(c) the timeaveraged size of the largest cluster observed for each configuration, $\left\langle S_{\max }\right\rangle_{t}$, as a function of density. For procedure 1, we see that for temperatures up to $2500 \mathrm{~K}$ the maximal 
cluster size $\left\langle S_{\max }\right\rangle_{t}$ observed remains constant at about 700 atoms (as in the solid state) down to a density of 0.01 atoms $/ \AA^{3}$ which corresponds to about $1 / 10$ of the density in the solid state. For these volume-temperature combinations, the average cluster size $\left\langle S_{\text {mean }}\right\rangle_{t}$ already begins to decrease indicating the presence of "gas molecules" in addition to the solid/liquid phase. Procedure 2 leads to the same behavior but again with a larger stability of the large clusters (encompassing nearly all atoms in the simulation cell) for low densities.

One should note that the curves for $\left\langle S_{\max }\right\rangle_{t}$ are nearly identical for $T \leq 4000 \mathrm{~K}$, although for low temperatures we are clearly dealing with a solid, while for temperatures exceeding $2000 \mathrm{~K}$ a melt is present (c.f. the analysis in subsection 3.3 and figure 3 a)), for both procedures. Similarly, we see in figure $3 \mathrm{~b}$ ) that the data points for $\left\langle N_{c l}\right\rangle_{t}$ for densities below 0.01 atoms $/ \AA^{3}$ also fall on the same curve for temperatures up to 3000 $\mathrm{K}$ for procedure 1. This suggests the interpretation that up to $4000 \mathrm{~K}$ we can easily distinguish between a condensed and a gaseous phase, which is probably no longer true above $4000 \mathrm{~K}$.

An interesting but hard to quantify observation in the stretched melt model is the fact that in the low density range $\left(\rho_{N}<0.01\right.$ atoms $\left./ \AA^{3}\right)$ we often find that the large clusters do not form compact objects. Instead, they appear to take on rather diffuse shapes, suggesting that we are either dealing with many smaller clusters or fragments that are more or less loosely connected to each other, or witness the slow compactification of a very large cluster from many small ones.

For temperatures exceeding $4000 \mathrm{~K}$, we find, for both procedures, that clusters of the size of the whole system are only present at very high densities, while the mean cluster size decreases much more rapidly with volume than even for temperatures in the range $2000-3000 \mathrm{~K}$. Clearly, the range of volumes where equilibrium between a condensed phase and a gaseous phase exists is shrinking, and the fluid consists of small clusters that inter-connect periodically.

More details are gained from the study of the actual cluster distribution. In figure 
4, we show for three characteristic temperatures, the likelihood of an atom to be part of a cluster of size $S_{c l},\left\langle L\left(S_{c l}\right)\right\rangle_{t}$ for both procedures. A binary distribution of $\left\langle L\left(S_{c l}\right)\right\rangle_{t}$ with peaks near $S_{c l}=1$ and $S_{c l}=N_{a t o m}$, indicates a condensed phase in equilibrium with a gaseous phase, while a function with a single peak corresponds to an essentially homogeneous fluid phase. We see that for procedure 1, a multi-cluster phase is found for all temperatures for a density below 0.01 atoms $/ \AA^{3}$, with some small amount of "gas" phase present already at higher densities and low temperatures. In contrast, procedure 2 yields essentially no gas phase for temperatures up to $3000 \mathrm{~K}$, and even at $4000 \mathrm{~K}$ some remnant of a condensed phase appears to be present for all densities.

Finally, an important aspect is the stability of the clusters and, for the large ones encompassing nearly the whole system, their viscosity. In order to estimate these quantities, we have computed the bond survival probabilities BSP between the atoms, and the diffusion coefficients $\mathrm{D}(\mathrm{V}, \mathrm{T})$.

Figure 6 shows the dependence of the bond survival probabilities BSP $\left(t_{w}=5 \cdot 10^{5}\right.$ MCC, $t_{o b s}=5 \cdot 10^{5} \mathrm{MCC}$ ) for Si-N and B-N on the densities and the temperature, for both procedures. For fixed temperature, all curves for procedure 1 exhibit a very flat minimum in the density range 0.1 atoms $/ \AA^{3}>\rho_{N}>0.01$ atoms $/ \AA^{3}$, where both the depth of the minimum and its breadth increase with temperature. For procedure 2, this effect is much less visible: for fixed temperature, the BSPs are nearly constant as a function of density.

Qualitatively, this can be understood as follows: For all temperatures, the high-density region has a reduced atom mobility such that a sizeable fraction of the bonds is going to survive on the time scale measured $(\approx 250 \mathrm{psec})^{5}$. Similarly, in the gaseous phase, the few bonds that still exist after a relaxation for a time $t_{w}=5 \cdot 10^{5} \mathrm{MCC}$ are likely to have survived for another time span of $t_{o b s}=5 \cdot 10^{5}$ MCC. $^{6}$ In-between, the condensed phase and the large clusters are going to exhibit the full malleability of the relaxed condensed

\footnotetext{
${ }^{5}$ For high enough pressures, we are dealing with a solid phase even at very high temperatures - there is no critical point in the solid/fluid transition.

${ }^{6}$ We would expect the free energy barriers associated with bond switches in the liquid to be much lower than those associated with the break-up of a small molecule or cluster.
} 
state, which is essentially independent of volume for quite a range of densities.

After comparing the $B S P$ obtained in this work with those of our studies of aging and the ergodic-non-ergodic transition[26, 20], we select $1 / e(\approx 0.3)$ and 0.8 as the critical values for the BSP: For $B S P<1 / e$, we are dealing with a liquid, and $B S P>0.8$ indicates a solid.

Finally, we turn to the diffusion constants shown in figure 7. Similar to the distinction between a glass and a (supercooled) liquid, we called the system an (amorphous) solid, if the diffusion coefficients $D(V, T)$ were below $0.01 \AA^{2} / \mathrm{MCC}$ (corresponding to about $\left.2 \cdot 10^{-3} \mathrm{~cm}^{2} / \mathrm{sec}\right)$. Using this approach, we note that the condensed amorphous phase remains solid up to about $1750 \mathrm{~K}$.

For comparison, we also performed heating experiments on a hypothetical crystalline $\mathrm{Si}_{3} \mathrm{~B}_{3} \mathrm{~N}_{7}$ solid phase[27], which was found to be stable up to $2500 \mathrm{~K}$. Apart from this difference in the melting/softening temperature, the thermodynamic properties computed when starting from the crystalline solid were the same as those for the amorphous solid. In the temperature range in-between one would expect the liquid to exhibit supercooling. Furthermore, we see a significant increase of $D(V, T)$ for all temperatures when lowering the density - here, the contribution of the gas phase to the average diffusion coefficient makes itself felt, of course.

\subsection{Construction of the (metastable) phase diagram}

From the results of the previous subsection 3.2, we can construct phase diagrams in the $(V, T)$ plane, shown in fig. 8. Both procedures yield very similar results for densities above 0.01 atoms $/ \AA^{3}$, and also for temperatures above $4000 \mathrm{~K}$. As expected, the differences are most visible in the low temperature-low density region. In order to decide, which of the two procedures produces the more accurate results in which region of the phase diagram, we have estimated the free energy for both procedures, using

$$
F^{(1,2)} \approx\langle E\rangle_{t}^{(1,2)}-T\langle S\rangle_{t}^{(1,2)}
$$


where $\langle E\rangle_{t}^{(1,2)}=\left\langle E_{k i n}\right\rangle_{t}^{(1,2)}+\left\langle E_{\text {pot }}\right\rangle_{t}^{(1,2)}=\left\langle E_{\text {pot }}\right\rangle_{t}^{(1,2)}+3 / 2 N k_{B} T$. For the entropic contribution $\langle S\rangle_{t}$, we employed a simple lattice gas approximation ${ }^{7}$

$$
S=k_{B} \ln \left[\frac{N_{l a t t} !}{\left\langle N_{c l}\right\rangle_{t} !\left(N_{l a t t}-\left\langle N_{c l}\right\rangle_{t}\right) !}\right]
$$

with

$$
N_{\text {latt }}=\frac{V}{\left\langle S_{\text {mean }}\right\rangle_{t} V_{\text {atom }}}=\frac{V\left\langle N_{c l}\right\rangle_{t}}{N_{\text {atom }} V_{\text {atom }}}=\frac{V}{V_{0}}\left\langle N_{c l}\right\rangle_{t}
$$

Since we are only interested in the approximate difference between the two systems, we did not include the entropic contributions due to the vibrations of the atoms belonging to the various clusters in the two procedures in this simple estimate, thereby tacitly assuming that the contributions of the vibrations can be taken to be approximately equal for the purpose of these (relatively rough) calculations.

Using equation 5 , we find that for $T \leq 3000 \mathrm{~K}, F^{(2)} \leq F^{(1)}$ (c.f. fig. 9). In particular, the low density region $\rho_{N}<0.01$ atoms $/ \AA^{3}$ appears to be better described by procedure 2. Thus, for this volume and temperature range, the system is in the condensed phase, with only a minute amount of gas phase present. On the other hand, for $T \geq 4000 \mathrm{~K}$, $F^{(1)} \leq F^{(2)}$, indicating that here in the low density region a gas-like cluster fluid is present that is separated from the condensed (liquid) phase by a two-phase co-existence region.

Figure 10 shows the final (metastable) phase diagram, where we have included the fact that the crystalline ternary solid is stable up to $2500 \mathrm{~K}$. We see that up to about $2500 \mathrm{~K}$, the system is a crystalline solid for all densities investigated (up to $2000 \mathrm{~K}$, the amorphous solid state would also be stable compared to the liquid). For $T \approx 1750-2500 \mathrm{~K}$, we find a "liquid-solid quasi-co-existence" region, i.e. the melt can be supercooled, at least on the time scales of our simulations, based on the analysis of the BSPs and the diffusion coefficients. Apart from technical aspects of the simulations, such as finite simulation times and system sizes, the reason for the existence of such a region is the occurrence of a glass transition in this temperature range between the liquid melt and the amorphous solid. Up to about $3000-4000 \mathrm{~K}$, we find the liquid state, with a small admixture of gas

\footnotetext{
${ }^{7}$ For $V \gg V_{0}$ and $\left\langle N_{c l}\right\rangle_{t} \gg 1,\langle S\rangle_{t} \approx\left\langle N_{c l}\right\rangle_{t} \ln \left(V / V_{0}\right)$.
} 
phase molecules. For even higher temperatures, the gaseous state prevails. Here, we also find a certain volume range, where both liquid and gas phase are present in substantial amounts. Extrapolating from our data, we estimate that this co-existence region should continue up to about $8000-9000 \mathrm{~K}$, resulting in a critical point at about $\rho_{c r} \approx 0.03$ atoms $/ \AA^{3}$ and $T_{c r} \approx 8500 \mathrm{~K}$.

\section{Discussion}

\subsection{Van der Waals-type model of the fluid region of the phase diagram}

In the preceding section, we have analyzed the cluster size distribution observed for the two simulation procedures. Next, we have used these distributions together with information about the bond survival probability and some simple estimates of the free energy to deduce a phase diagram for the ternary region of the system $\mathrm{Si}_{3} \mathrm{~B}_{3} \mathrm{~N}_{7}$.

By extrapolation from the phase diagram, we estimated that the system should possess a critical point in the liquid-gas region at about $T_{c r} \approx 8500 \mathrm{~K}$ and $\rho_{c r} \approx 0.03$ atoms $/ \AA^{3}$. Another way to gain an estimate for the location of this critical point is to attempt to fit the $p(V, T)$ curves in the high-temperature regime to an empirical equation of state. As we noted in subsection 3.3, procedure 1 appears to lead to somewhat more realistic values for this region $(T \geq 4000 \mathrm{~K})$. Thus, we have performed a fit of the simulated data of procedure 1 to a van der Waals equation,

$$
\left(p+p_{0}(V, T)\right)(V-b(V, T))=N_{a t o m} k_{B} T
$$

where $p_{0}$ is commonly assumed to depend on volume via $p_{0}=a / V^{2}$, and $b$ is identified with the atomic volume $b=N_{\text {atom }} V_{\text {atom }}=V_{0}$.

The fit works best for high and low densities, while the negative pressures in the intermediate density region are not well described. From the fit parameters, we can now calculate the critical values for pressure, temperature and density, $p_{c r} \approx 1.3 \mathrm{GPa}$, 
$T_{c r} \approx 8000 \mathrm{~K}$ and $\rho_{c r} \approx 0.032$ atoms $/ \AA^{3}$, respectively. This is in satisfactory agreement with our rough estimate from the phase diagram.

Of course, we cannot expect the system to really obey the equation of state of a van der Waals fluid, even apart from computational issues. The reason is that the mean field picture that justifies eq. 8 by assuming essentially identical monoatomic featureless fluid particles interacting with the mean field created by the other particles in the system, qualitatively fails for $\mathrm{Si}_{3} \mathrm{~B}_{3} \mathrm{~N}_{7}$. As we saw in subsection 3.2, even at very high temperatures and large volumes, we still observe non-neglible atom-atom interactions that result in the presence of small clusters of atoms in the fluid phase. Of course, these will contribute to the free energy of the system, and, in the context of the van der Waals picture, result in terms in $p_{0}$ that are only very weakly dependent on volume, $p_{0} \approx a_{1} / V^{\alpha}+a_{2} / V^{2}$, with $1<\alpha<2$.

On the other hand, the mean field picture is moderately realistic for the state of the fluid at high densities, where most atoms are part of larger clusters, and thus all atoms experience the same "average" interaction over the observation time. We note that, on this level of sophistication, this also includes the solid state of the system. As we have seen, distinguishing between the liquid and the solid state is more subtle at this level, of course, and requires the introduction of time-dependent quantities such as the bond survival probability, or the mean square displacement.

Another issue we should mention is the fact that quite a number of data points are in a region, where in the van der Waals picture we would expect the system to be thermodynamically unstable. In our simulations, this makes itself felt as a phase separation into e.g. a liquid-plus-gas or a solid-plus-gas mixture.

\subsection{Metastability of the phase diagram: Phase segregation, and effects due to finite size/time and model potential}

We have repeatedly indicated that the phase diagram we present is an intrinsically metastable one, and strictly only applies for the ternary region of the space of $\mathrm{Si} / \mathrm{B} / \mathrm{N}$ 
compounds for one particular composition. The reason for this caveat is threefold:

For one, the time scales of the simulations we are able to perform ${ }^{8}$ are several orders of magnitude below those needed for the system to reach full thermodynamic equilibrium. One consequence of this is that we never observe a crystalline variant of $\mathrm{Si}_{3} \mathrm{~B}_{3} \mathrm{~N}_{7}$ nor the formation of crystalline binary phases such as $\mathrm{Si}_{3} \mathrm{~N}_{4}$ or $\mathrm{BN}$ when starting from e.g. a supercooled stretched-melt configuration. Furthermore, except at high temperatures, we did not reach convergence of the two simulation procedures to the same equilibrium state $^{9}$.

While we were able to perform some simple (free energy based) estimates, which procedure was more appropriate for which region of the phase diagram, we were not assured to have reached equilibrium in either one. In the solid state at low temperatures, all configurations observed were amorphous structures, except when starting with a crystalline one, of course. As mentioned above, we also computed the energy of several hypothetical crystalline structure candidates, and also the energies of the binary crystalline compounds $\beta$-Si ${ }_{3} \mathrm{~N}_{4}$ and h-BN. We find that the energetically lowest state corresponds to a weighted mixture of $\beta-\mathrm{Si}_{3} \mathrm{~N}_{4}$ and h-BN, followed by the ternary crystalline modifications of $\mathrm{Si}_{3} \mathrm{~B}_{3} \mathrm{~N}_{7}$.

We note that the ternary crystalline state does melt at about $2500 \mathrm{~K}$. Thus, including this state does not influence the shape of the phase diagram by much, except at very high densities. Regarding the $\mathrm{BN}$ and $\mathrm{Si}_{3} \mathrm{~N}_{4}$ phase separation, we note that this would (in the context of the simulations using a finite simulation cell) correspond to an energetically unfavorable interface, which would prevent us from observing such a phase separation.

In order to investigate this issue, we have stacked two infinite (in the $(x, y)$-direction) slabs of $\beta$ - $\mathrm{Si}_{3} \mathrm{~N}_{4}$ and $\mathrm{h}$-BN along the $z$-direction of the periodically repeated simulation cell $(a=13.04 \AA, b=15.08 \AA, c \text { variable })^{10}$, and allowed the system to relax at constant pressure. In order to achieve an optimally matched interface, we have first scanned the

\footnotetext{
${ }^{8}$ The simulations presented took the equivalent of about 5 years on an $3.2 \mathrm{GHz}$ processor.

${ }^{9}$ Many other criteria did appear to indicate equilibrium at temperatures above $2000 \mathrm{~K}$, however, such as the potential energy as function of time.

${ }^{10}$ The hexagonal unit cells had been transformed to the corresponding orthorhombic setting.
} 
energy of about $10^{5}$ relative positions of the two slabs (in the $(x, y)$-plane), followed by a NpT-relaxation of the arrangements with the lowest energy. We find that at $T=500$ $\mathrm{K}$, the average energy of the two-slab configuration decreases with increasing thickness of the slabs (proportional to the cell length $c$ ), and reaches the value of the phase-separated bulk h-BN-plus- $\beta-\mathrm{Si}_{3} \mathrm{~N}_{4}$ system for infinite thickness (c.f. fig. 11).

We can explicitly calculate the interface energy $\sigma_{i n t}=(n / 2) m$, where $m$ is the slope of the curve depicting the energy of the slab vs. $1 / c$ in fig. 11 and $n \approx 0.1 \AA^{-3}$ is the number density. We find $m=2.19 \mathrm{eVA}$ and $\sigma_{i n t} \approx 0.11 \mathrm{eV} / \AA^{2}$.

Figure 11 also yields critical slab thicknesses $d_{\text {slab }}=c / 2$ of $d_{\text {slab }}^{a m o} \approx 10 \AA$ and $d_{\text {slab }}^{\text {cry }} \approx 50$ $\AA$, where the energy of the slab configuration equals the energy of the ternary amorphous and crystalline phase, respectively. Even taking into account that nano-sized crystal fragments have larger surfaces than semi-infinite slabs (and thus higher interface energies per atom), our results suggest that one would need nano-crystallites with diameters below $d_{c f}^{a m o}=3 d_{s l a b}^{a m o} \approx 30 \AA$ to achieve a stable homogeneous sinter of the amorphous phase at low temperatures. ${ }^{11}$ This agrees very well with the observation that the synthesis of a$\mathrm{Si}_{3} \mathrm{~B}_{3} \mathrm{~N}_{7}$ via sintering a mixture of $\mathrm{BN}$ and $\mathrm{Si}_{3} \mathrm{~N}_{4}$ microcrystallites (diameter about 500 $\mathrm{nm})$ has failed[6]. Similarly, the ternary crystalline phase is preferred against a mixture of crystallites of the binary phases up to diameters of $d_{c f}^{c r y}=3 d_{s l a b}^{c r y} \approx 150 \AA$. In this context, we note that the size of our simulation cell in the solid state $(\approx 19 \AA)$ lies somewhere between $d_{\text {slab }}^{a m o}$ and $d_{s l a b}^{c r y}$. Thus, even at equilibrium a phase separation into the binary end phases would not be clearly preferred in the simulations.

This points to the second complication we face: the finite size of the system. While simulations of very large e.g. Lennard-Jones and soft-sphere systems have been performed in the past [31], $\mathrm{Si}_{3} \mathrm{~B}_{3} \mathrm{~N}_{7}$ is considerably more complicated, and thus we were limited in the system size we could treat. As is well-known, finite size effects, in particular the existence

\footnotetext{
${ }^{11}$ In earlier work[14], we have proposed a model for a-Si ${ }_{3} \mathrm{~B}_{3} \mathrm{~N}_{7}$ consisting of sintered crystal fragments of diameter $\approx 5-10 \AA$. The energy difference between this model and the phase segregated material, $\approx 0.2 \mathrm{eV} /$ atom, agrees satisfactorily with a rough estimate of the interface energy of the crystal fragment model, $\approx 0.3 \mathrm{eV} /$ atom.
} 
of non-negligible surfaces of the condensed phase and the formation of interfaces between condensed and gaseous phases, bedevil the computer experimentalist[31]. Clearly, this is a reason behind the appearance of negative pressures at intermediate densities. In order to gain some insight into the effects of finite size, we have repeated the calculations for a system of half the size $\left(N_{\text {atom }}=351\right)$, for both procedures. In order to save some computation time, we have performed these calculations on a wider grid of volume values, but for the full temperature range. We find no qualitative change from the results in the larger system.

Finally, there is the question of the potential we have employed to model the interactions among the atoms. While it is well-suited to represent hetero-atom interactions between different types of atoms, it does not properly apply to the elements themselves, i.e. for neither elementary $\mathrm{Si}$ or $\mathrm{B}$ nor $\mathrm{N}_{2}$ molecules can one compute the correct bond energies that would allow a comparison with the binding energies in the binary and ternary compounds. In particular, our results must exclude the formation of $\mathrm{Si}, \mathrm{B}$ or $\mathrm{N}_{2}$ vapor. Especially the latter is sorely missed, since it might most easily form at high temperatures and low pressures, opening a possibly important route to decomposition of the ternary compound. From the point of view of the experimentalist, mapping the phase diagram outlined in this work should therefore presumably take place in a nitrogen saturated atmosphere.

A very rough estimate of the pressure needed to suppress the evolution of $\mathrm{N}_{2}$ from the condensed phase at relatively low temperatures can be derived from comparing the free energies,

$$
F\left(\mathrm{Si}_{3} \mathrm{~B}_{3} \mathrm{~N}_{7}\right) \approx E\left(\mathrm{Si}_{3} \mathrm{~B}_{3} \mathrm{~N}_{7}\right) \approx E\left(\mathrm{Si}_{3} \mathrm{~N}_{4}\right)+E(\mathrm{BN})
$$

and

$$
F\left(\mathrm{Si}, \mathrm{B}, \mathrm{N}_{2}\right) \approx E(\mathrm{Si}, \mathrm{B})+E\left(\mathrm{~N}_{2}\right)-k_{B} T \ln g\left(\mathrm{~N}_{2}\right)
$$

where $g\left(\mathrm{~N}_{2}\right)$ is the number of ways $\mathrm{N}_{2}$ molecules can be placed in a cubical box of volume $V$. This yields

$$
p_{\text {limit }}(T) \approx \rho_{\text {limit }} k_{B} T
$$


with

$$
\rho_{\text {limit }} \approx 0.1 \cdot \exp \left[\left(E\left(\mathrm{Si}_{3} \mathrm{~N}_{4}\right)+E(\mathrm{BN})-E(\mathrm{Si}, \mathrm{B})-E\left(\mathrm{~N}_{2}\right)\right) /\left(N\left(\mathrm{~N}_{2}\right) k_{B}\right) T\right]
$$

in units of atoms $/ \AA^{3}$. Taking these data from experiment, we find $p_{\text {limit }} \approx 0.16 k_{B} T$. In particular, $p_{\text {limit }}(2000 \mathrm{~K}) \approx 4 \mathrm{GPa}$.

\subsection{Complementarity of the approach to other simulation pro- cedures}

A cluster-size analysis similar in some aspects to the work presented here, has been performed in the past[32] for the derivation of the phase diagrams of many elements, such as elemental sodium. There, an analytical mean-field expression of interacting clusters was investigated and the corresponding Gibbs free energy was minimized with respect to the average cluster size and the overall density. For metals, it was found that the typical cluster sizes were very small $\left(N_{c l}=1-3\right),{ }^{12}$ while for group 4 elements that exhibit a stronger tendency towards network formation, the melt contained clusters of size up to ten close to the freezing temperature.

Much of the more recent computational work in the literature has focused on the liquid-gas region and to a lesser extent on the solid-gas region, where large computer simulations have been performed. In particular, Lennard-Jones type[33, 34, 35] and hardand soft-sphere systems[36] have been investigated. However, these types of systems are not well suited to model network forming systems that can exhibit a wide variety of clusters in the liquid-gas region of the phase diagram. This caveat also extends to Gibbs ensemble simulations of phase-equilibration[37], since the exchange of atoms between the liquid (not to mention the solid ${ }^{13}$ ) and gaseous phases is far from trivial in a system like $\mathrm{Si}_{3} \mathrm{~B}_{3} \mathrm{~N}_{7}$.

\footnotetext{
${ }^{12}$ In the super-cooled state, the cluster size could increase considerably.

${ }^{13} \mathrm{An}$ interesting extension to Gibbs ensemble simulations that cover solid-vapor phase equilibria has recently been proposed [38].
} 
This concern similarly applies to the use of alternative sampling techniques such as parallel or extended sampling of configuration space (see e.g. [23, 24]) used to compute the stability of phases, the calculation of the free energy via density of states methods $[23,39,40,41,42,43,44,45]$ or multicanonical analysis techniques $[24,46,47,48,49,50]$, all of which may be used to refine the precise location of the phase boundary. Similarly, the computational effort would be extreme for various umbrella sampling techniques employed to evaluate free energy differences such as thermodynamic perturbation[51], thermodynamic integration[52] and finite-time variation[53] (even if one uses optimized thermodynamic paths[54]), or for the use of transition path sampling[55, 56, 57, 58, 59] and the so-called metadynamics $[60,61,62]$ in the analysis of the kinetic and thermodynamic stability of different phases. Nevertheless, there exist studies dealing with systems where more complex interactions than simple isotropic two-body potentials are involved; e.g. the investigation of the kinetics of freezing[63] and boiling[64] of water, or the computation of (part of) the phase diagram of carbon on ab initio level using thermodynamic integration[65]. Here, we find some similarities to the behaviour of $\mathrm{Si}_{3} \mathrm{~B}_{3} \mathrm{~N}_{7}$; e.g. the shapes of the clusters that are observed in the liquid-gas transition of $\mathrm{H}_{2} \mathrm{O}$ bear some similarity to the ones we find in the liquid-gas co-existence region of the phase diagram of $\mathrm{Si}_{3} \mathrm{~B}_{3} \mathrm{~N}_{7}$.

Of course, in the future, investigations using refined techniques such as the ones mentioned above should become feasible even for systems as complex as $\mathrm{Si}_{3} \mathrm{~B}_{3} \mathrm{~N}_{7}$. However, the approach presented here at the example of $\mathrm{Si}_{3} \mathrm{~B}_{3} \mathrm{~N}_{7}$ can serve as a first step towards an efficient methodology to compute the general features of the full solid/liquid/gas phase diagram of complex multinary systems. The proposed combination of a sequence of computer experiments starting from several physically reasonable initial configurations (quite similar to the way one often selects appropriate starting configurations for transition path sampling) and a set of well-crafted complementary analysis techniques for the a-posteriori determination of the states of matter one "encounters" during the computer simulations and these states' characterization by an appropriate indicator, is quite fast and easily 
controllable. Thus, our approach can be used for an efficient scan of the phase diagram of an unknown system (or one with only partly known thermodynamic behaviour) over a wide range of temperatures and pressures (or volumes). While this method lacks the quantitative precision of other techniques, the sketch of the global phase diagrams it produces can serve as a guide to the use of more refined but computationally more expensive procedures. In this sense, the approach presented here is complementary to alternative sampling methods such as multi-histogram sampling, (weighted) density of states sampling, metadynamics and transition path sampling, where one usually requires at least some rough idea, in what temperature/pressure range the transitions between two phases occur.

\section{$5 \quad$ Summary}

Starting with two complementary initial configurations, a 'stretched melt' and a 'free block' configuration of amorphous and (hypothetical) crystalline $\mathrm{Si}_{3} \mathrm{~B}_{3} \mathrm{~N}_{7}$ in periodically repeated simulations cells over a large range of densities, we have performed NVTsimulations for temperatures ranging from 250 to $7000 \mathrm{~K}$. We have studied the resulting time-averaged cluster distributions which, together with the bond survival probabilities and the diffusion constants, allowed us to identify the solid, liquid and gaseous regions of the metastable phase diagram as function of volume and temperature. Fitting a van der Waals equation of state to the fluid phase region of the phase diagram, we determined the critical point of the system to occur at about $8500 \mathrm{~K}$ and $1.3 \mathrm{GPa}$. By studying slabs of various thicknesses, we found that in the solid state a phase segregation into the binary phases h-BN and $\beta-\mathrm{Si}_{3} \mathrm{~N}_{4}$ would be favored over the (hypothetical) ternary crystalline phase for system sizes exceeding $150 \AA$. In addition, the calculations showed that the generation of amorphous a- $\mathrm{Si}_{3} \mathrm{~B}_{3} \mathrm{~N}_{7}$ via sintering of h-BN and $\beta$ - $\mathrm{Si}_{3} \mathrm{~N}_{4}$ crystallites would require the use of nanometer-size crystal fragments, explaining the failure of sintering experiments with micron-size particles. 


\section{Acknowledgments}

Funding was kindly provided by the DFG via SFB408. 


\section{References}

[1] M. Jansen, B. Jäschke, and T. Jäschke. Amorphous Multinary Ceramics in the SiB-N-C System, volume 101 of Structure 65 Bonding, pages 138-191. Springer-Verlag, Berlin, 2002.

[2] D. Seyferth and H. Plenio. J. Am. Ceram. Soc., 73:2131, 1990.

[3] H. P. Baldus, O. Wagner, and M. Jansen. In M. J. Hampden-Smith, W. G. Klemperer, and C. J. Brinker, editors, Mater. Res. Soc. Symp. Proc., volume 271, pages 821-826. Mat. Res. Soc., Warrendale, 1992.

[4] K. Su, E.E. Remsen, G.A. Zank, and L.G. Sneddon. Chem. Mater., 5:547, 1993.

[5] O. Funayama, T. Kato, Y. Tashiro, and T. Isoda. J. Am. Ceram. Soc., 76:717, 1993.

[6] M. Jansen and P. Baldus. Angew. Chem. Int. Ed. Engl., 36:328-344, 1997.

[7] P. Baldus, M. Jansen, and D. Sporn. Science, 285:699-703, 1999.

[8] F. Aldinger, M. Weinmann, and J. Bill. Pure Appl. Chem., 70:439, 1998.

[9] D. Srivastava, E.N. Duesler, and R.T. Paine. Eur. J. Inorg. Chem., 6:855, 1998.

[10] R. Riedel, A. Kienzle, W. Dressler, L. Ruwisch, J. Bill, and F. Aldinger. Nature, 382:796, 1996.

[11] M. Jansen, J. C. Schön, and L. van Wüllen. Angew. Chem. Int. Ed., 45(26):42444263, 2006.

[12] R.M. Hagenmayer, U. Müller, C. J. Benmore, J. Neuefeind, and M. Jansen. J. Mater. Chem., 9:2865, 1999.

[13] U. Müller, W. Hoffbauer, and M. Jansen. Chem. Mater., 12(8):2341-2346, 2000. 
[14] A. Hannemann, J. C. Schön, C. Oligschleger, and M. Jansen. In B. Müller, editor, Proceedings of DGK-workshop on 'Struktur und Eigenschaften nichtkristalliner Materialien - Meßdaten und Strukturmodelle' (Wolfersdorf Sept. 1999); also condmat/0001319. Univ. Jena, Jena, 1999.

[15] A. Hannemann, J.C. Schön, H. Putz, T. Lengauer, and M. Jansen. Phys. Rev. B, $70: 144201,2004$.

[16] L. van Wüllen, U. Müller, and M. Jansen. Chem. Mater., 12(8):2347-2352, 2000.

[17] J. C. Schön, A. Hannemann, and M. Jansen. J. Phys. Chem. B, 108:2210-2217, 2004.

[18] A. Hannemann, J.C. Schön, and M. Jansen. J. Mater. Chem., 15:1167-1178, 2005.

[19] A. Hannemann, J.C. Schön, and M. Jansen. Phil. Mag., 85:2621-2639, 2005.

[20] A. Hannemann, J.C. Schön, M. Jansen, and P. Sibani. J. Phys. Chem. B, 109:1177011776, 2005.

[21] D.P. Landau and K. Binder. A Guide to Monte Carlo Simulations in Statistical Physics. Cambridge University Press, Cambridge, 1 edition, 2000.

[22] W. Kob. In J.L. Barrat, M. Feigelmann, and J. Kurchan, editors, Slow Relaxation and Nonequilibrium Dynamics in Condensed Matter, volume Session LXVII of Les Houches 2002 Summer School . Les Houches Summer School, World Scientific Singapore, 2002. see also cond-mat/0212344.

[23] M. Mueller and J. J. DePablo. In M. Ferrario, G. Ciccotti, and K. Binder, editors, Computer Simulations in Condensed Matter: From Materials to Chemical Biology, pages 65-123. Springer, New York, 2006.

[24] N. Wilding. In M. Ferrario, G. Ciccotti, and K. Binder, editors, Computer Simulations in Condensed Matter: From Materials to Chemical Biology, pages 40-64. Springer, New York, 2006. 
[25] M. Gastreich, C.M. Marian, and J.D. Gale. Phys. Rev. B, 62:3117-3123, 2000.

[26] J. C. Schön, A. Hannemann, G. Sethi, M. Jansen, P. Salamon, R. Frost, and L. Kjeldgaard. Proc. XXIII Workshop on structure and kinetics of nucleation and crystallization in non-crystalline materials, Jena September 2002; also as: condmat/0212279, 2002.

[27] J. C. Schön and M. Jansen. Angew. Chem. Int. Ed. Eng., 35:1286-1304, 1996.

[28] M. P. Allen and D. J. Tildesley. Computer Simulations of Liquids. Clarendon Press Oxford, 1987.

[29] T. Cormen, C. Leiserson, and R. Rivest. Introduction to Algorithms. MIT Press Boston McGraw Hill New York, 1990.

[30] L. Verlet. Phys. Rev., 159(1):98-103, 1967.

[31] D. Frenkel and B. Smit. Understanding Molecular Simulation From Algorithms to Applications. Academic Press, San Diego, $2^{\text {nd }}$ edition, 2002.

[32] J.C. Schön. Phd-thesis, Massachusetts Institute of Technology, July 1988.

[33] J.K. Johnson, J.A. Zollweg, and K. Gubbins. Mol. Phys., 78(3):591-618, 1993.

[34] M.R. Hitchcock and C.K. Hall. J. Chem. Phys., 110(23):11433-11444, 1999.

[35] M.A. Barroso and A.L. Ferreira. J. Chem. Phys., 116(16):7145-7150, 2002.

[36] R. Agrawal and D. A. Kofke. Mol. Phys., 85(1):43-59, 1995.

[37] A. Z. Panagiotopolus, N. Quirke, M.R. Stapleton, and D.J. Tildesley. Mol. Phys., 63:527-543, 1987.

[38] B. Chen, J.I. Siepmann, and M.L. Klein. J. Phys. Chem. B, 105:9840-9848, 2001.

[39] G. M. Torrie and J. P. Valleau. J. Comp. Phys., 23:187, 1974. 
[40] G. M. Torrie and J. P. Valleau. Chem. Phys. Lett., 28:578, 1977.

[41] J. Lee. Phys. Rev. Lett., 71:211-214, 1993.

[42] F. Wang and D. P. Landau. Phys. Rev. Lett., 86:2050, 2001.

[43] F. Wang and D. P. Landau. Phys. Rev. E, 64:056101, 2001.

[44] Q. Yan, R. Faller, and J. J. dePablo. J. Chem. Phys., 116:8745, 2002.

[45] Q. Yan and J. J. DePablo. Phys. Rev. Lett., 90:035701, 2003.

[46] R. H. Swendsen and A. M. Ferrenberg. Phys. Rev. Lett., 61:2635, 1989.

[47] R. H. Swendsen and A. M. Ferrenberg. Phys. Rev. Lett., 63:1195, 1993.

[48] B. A. Berg and T. Neuhaus. Phys. Lett. B, 267:249, 1991.

[49] B. A. Berg and T. Neuhaus. Phys. Rev. Lett., 68:9, 1992.

[50] G. R. Smith and A. D. Bruce. Phys. Rev. E, 53:6350, 1996.

[51] R. Zwanzig. J. Chem. Phys., 22:1420-1426, 1954.

[52] J. G. Kirkwood. J. Chem. Phys., 3:300-313, 1935.

[53] M. Watanabe and W. P. Reinhardt. Phys. Rev. Lett., 65:3301-3304, 1990.

[54] J. C. Schön. J. Chem. Phys., 105:10072-10083, 1996.

[55] P. G. Bolhuis, C. Dellago, and D. Chandler. Faraday Discuss., 110:421-436, 1998.

[56] C. Dellago, P. Bolhuis, F. S. Csajka, and D. Chandler. J. Chem. Phys., 108:1964, 1998.

[57] P. G. Bolhuis, D. Chandler, C. Dellago, and P. L. Geissler. Ann. Rev. Phys. Chem., 53:291-318, 2002.

[58] D. Zahn and S. Leoni. Phys. Rev. Lett., 92:250201, 2004. 
[59] C. Dellago, P. Bolhuis, and P. L. Geissler. In M. Ferrario, G. Ciccotti, and K. Binder, editors, Computer Simulations in Condensed Matter: From Materials to Chemical Biology, page 124. Springer, New York, 2006.

[60] R. Martonak, A. Laio, and M. Parrinello. Phys. Rev. Lett., 90:75503, 2003.

[61] R. Martonak, A. Laio M. Bernasconi, C. Ceriani, P. Raiteri, F. Ziploi, and M. Parrinello. Z. Kristallogr., 220:489-498, 2005.

[62] G. Bussi, A. Laio, and M. Parrinello. Phys. Rev. Lett., 96:090601, 2006.

[63] I. Ohmine, M. Matsumoto, and S. Saito. Nature, 416:409, 2002.

[64] D. Zahn. Phys. Rev. Lett., 93:227801, 2004.

[65] X. Wang, S. Scandolo, and R. Car. Phys. Rev. Lett., 95:185701, 2005. 


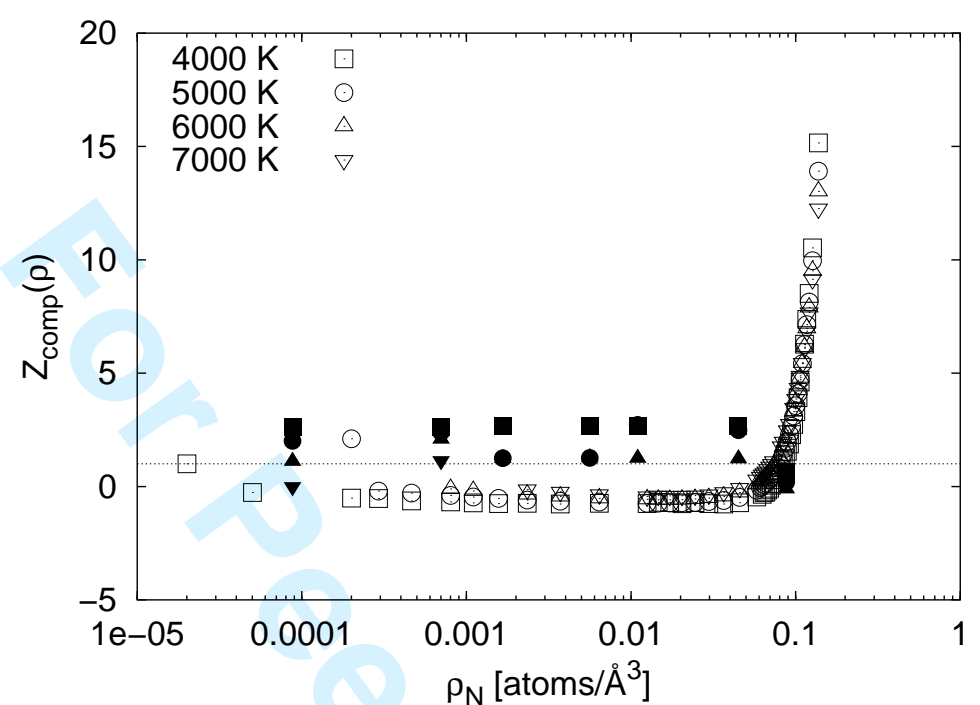

(a) Compression factors

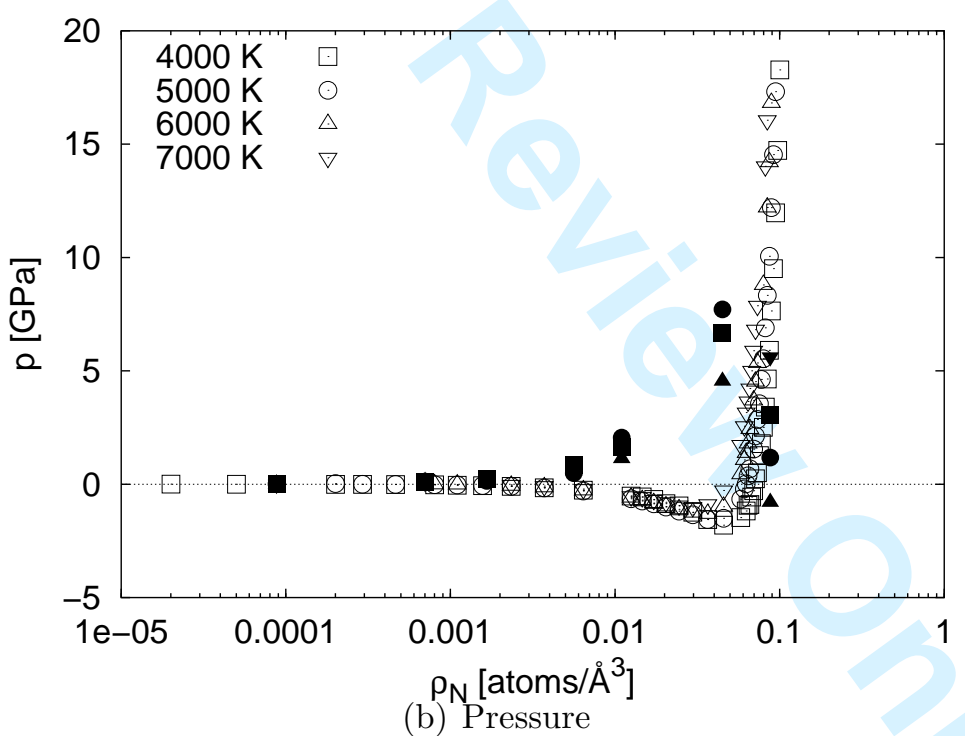

Figure 1: Dependence of the compression factor $Z\left(\rho_{N}\right)=\frac{p \cdot V}{N k_{B} T}$ and the pressure p on the number density $\rho_{N}$ for procedures 1 (open symbols) and 2 (filled symbols) for selected isotherms. 


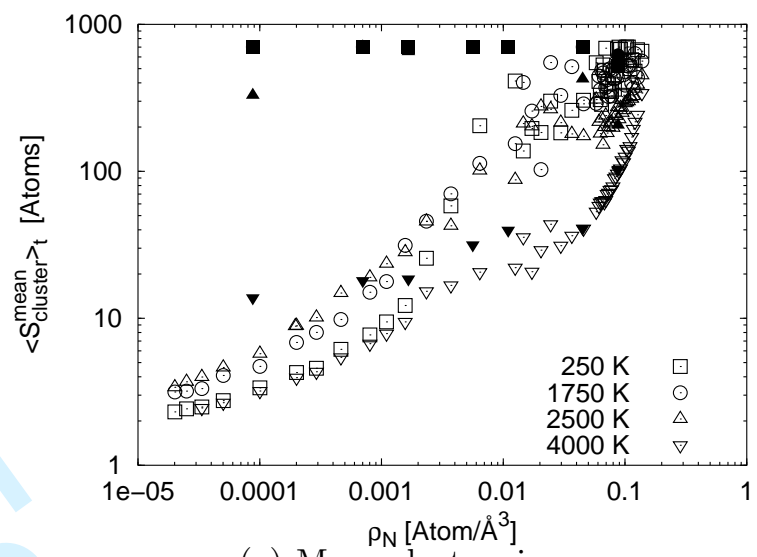

(a) Mean cluster size

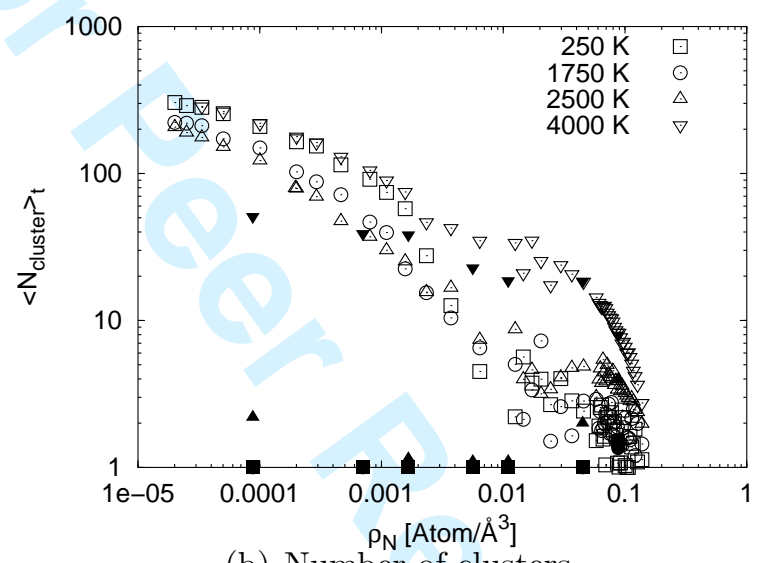

(b) Number of clusters

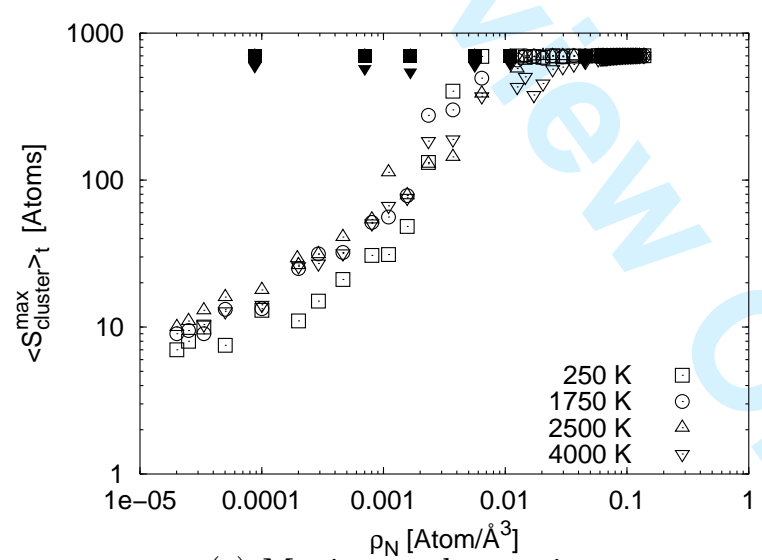

(c) Maximum cluster size

Figure 2: Dependence of the mean cluster size $\left\langle S_{\text {cluster }}^{\text {mean }}\right\rangle$, the number of clusters $N_{\text {cluster }}$ and the maximum cluster size $\left\langle S_{\text {cluster }}^{\max }\right\rangle$ on the number density $\rho_{N}$ for selected isotherms calculated from configurations generated by procedure 1 (open symbols) and procedure 2 (filled symbols) 

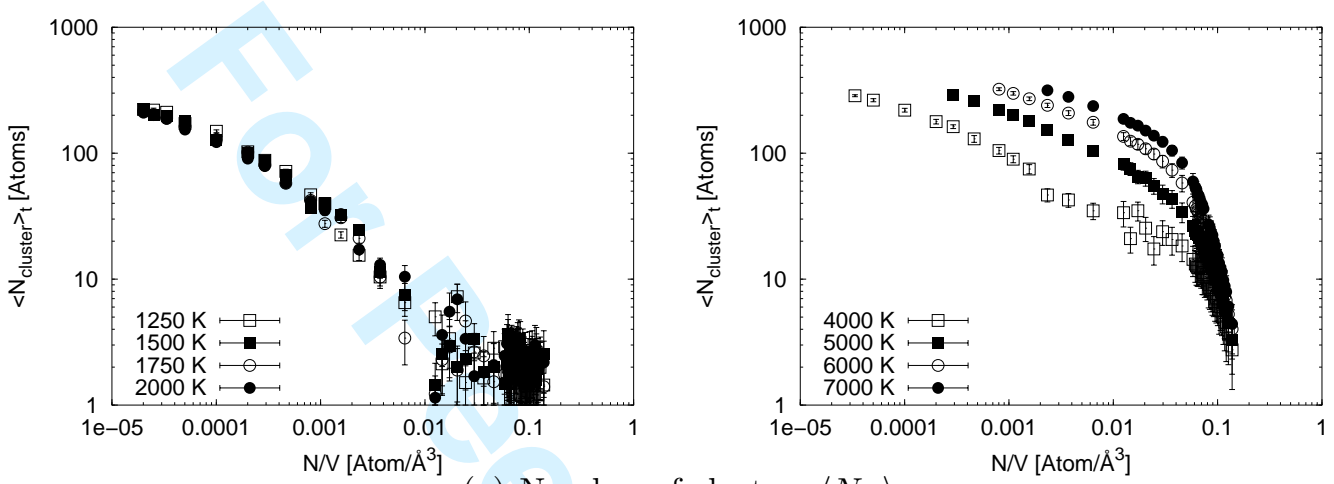

(a) Number of clusters $\left\langle N_{c l}\right\rangle$
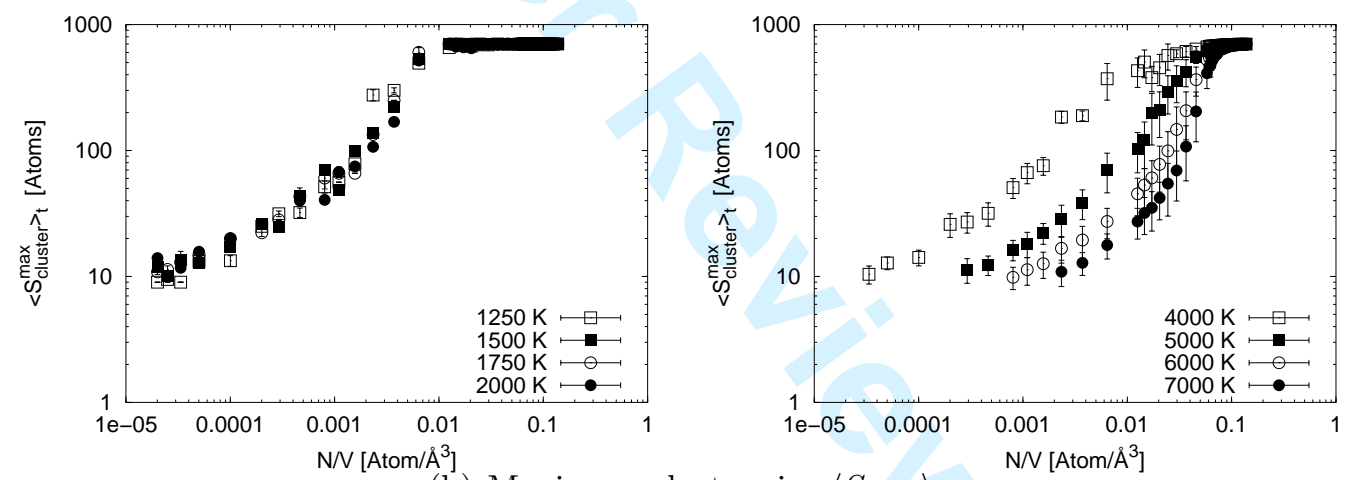

(b) Maximum cluster size $\left\langle S_{\max }\right\rangle_{t}$

Figure 3: Dependence of time averaged cluster distribution properties on the number density $N / V=\rho_{N}$ for a detailed set of temperatures given (in $\mathrm{K}$ ) in the legends. The number densities $\rho_{N}$ are given in atoms $/ \AA^{3}$. The properties were calculated from configurations generated by procedure 1 . 

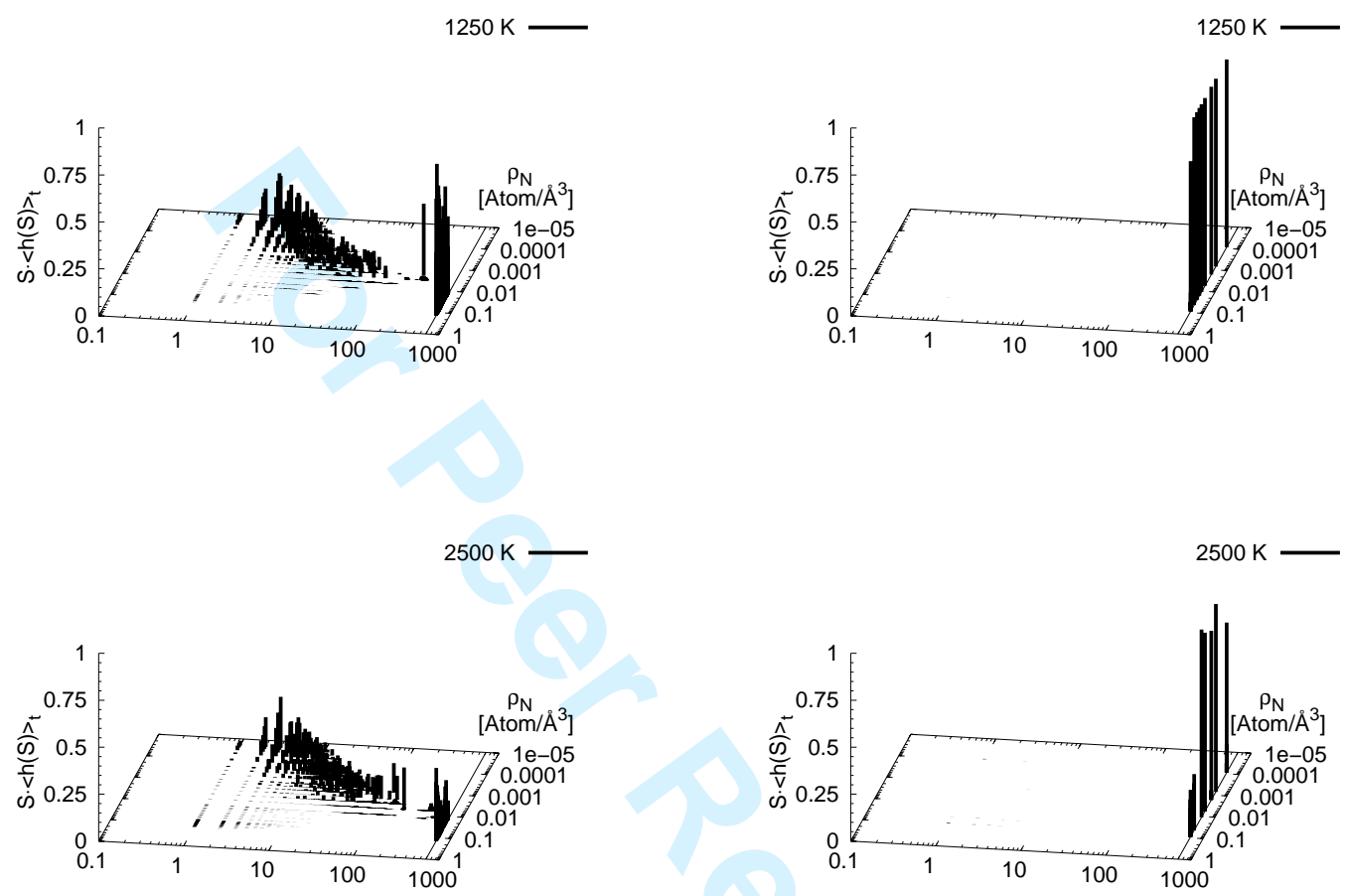

$5000 \mathrm{~K}$

$5000 \mathrm{~K}$

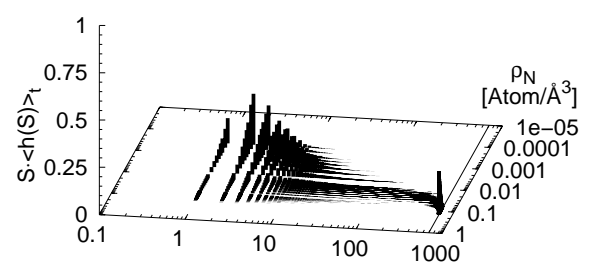

(a) Procedure 1

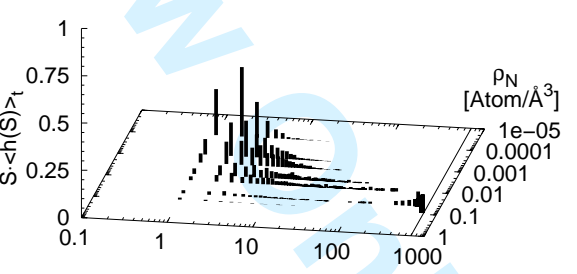

(b) Procedure 2

Figure 4: Dependence of the distribution of the mean number of atoms $S \cdot\langle h(S)\rangle$ inside a cluster of size $S_{\text {cluster }}$ on the number density $\rho_{N}=N / V$ for temperatures $T=1250,2500$ and $5000 \mathrm{~K}$. 


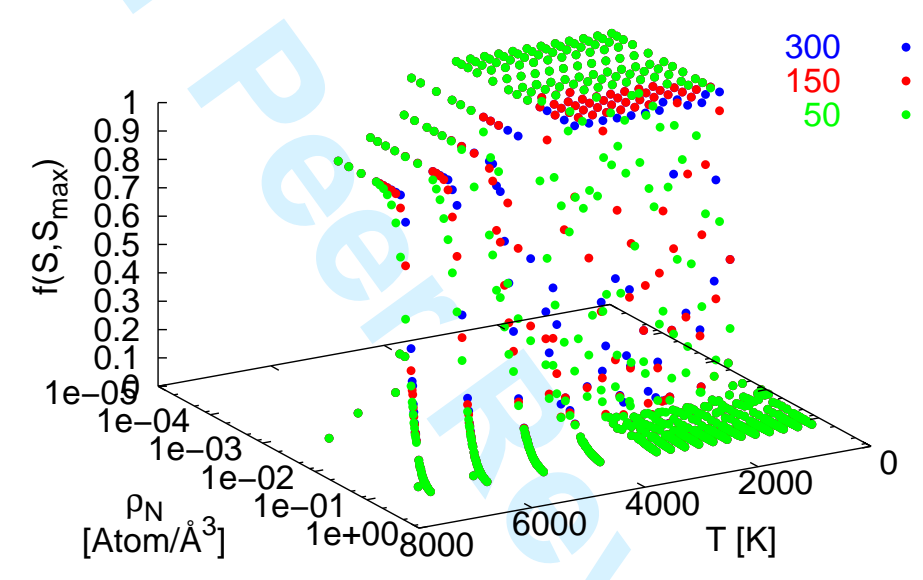

Figure 5: Dependence of fraction of atoms $f\left(S, S^{*}\right)$ defined by equation 4 on the number density $\rho_{N}$ (given in Atoms $/ \AA^{3}$ ) and the temperature $\mathrm{T}$ (given in $\mathrm{K}$ ) for three different choices for maximum cluster size $S^{*}$ employed in the calculation of $f\left(S, S^{*}\right)$. Data shown for configurations generated from procedure 1. 


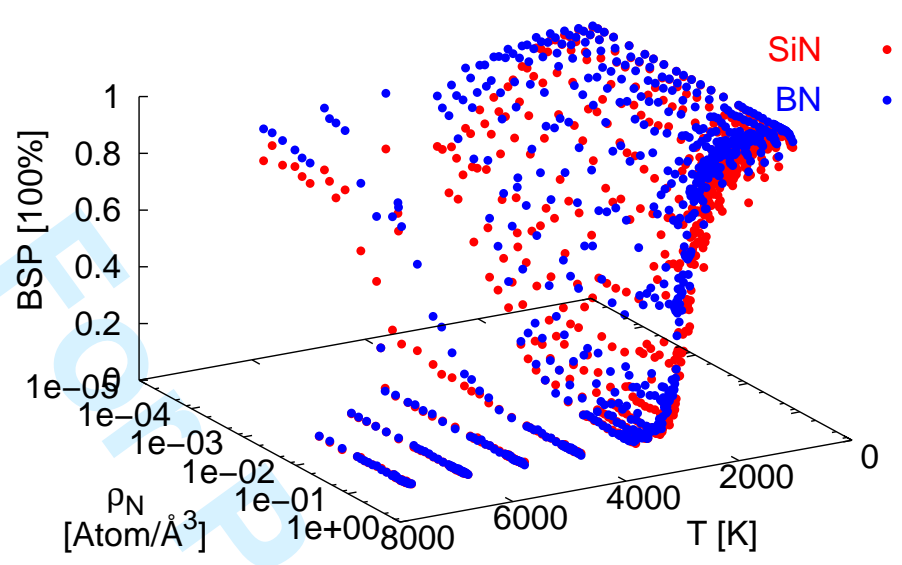

(a) Procedure 1

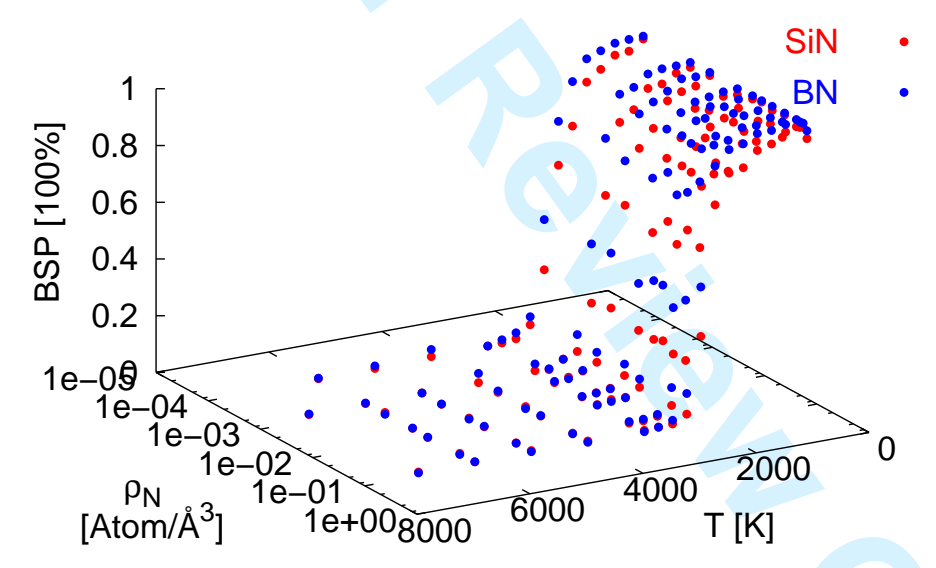

(b) Procedure 2

Figure 6: Temperature and density dependence of the time averaged bond survival probabilities BSP for silicon nitrogen (red) and boron nitrogen bonds (blue). The temperature is given in $\mathrm{K}$ and the densities are given in Atoms $/ \AA^{3}$. The time averages were calculated over the last $2 \cdot 10^{4} \mathrm{MCC}$ observation time steps and the BSP were calculated with respect to a waiting $t_{w}=5 \cdot 10^{5} \mathrm{MCC}$. The total observation time was $t_{o b s}=5 \cdot 10^{5} \mathrm{MCC}$. 


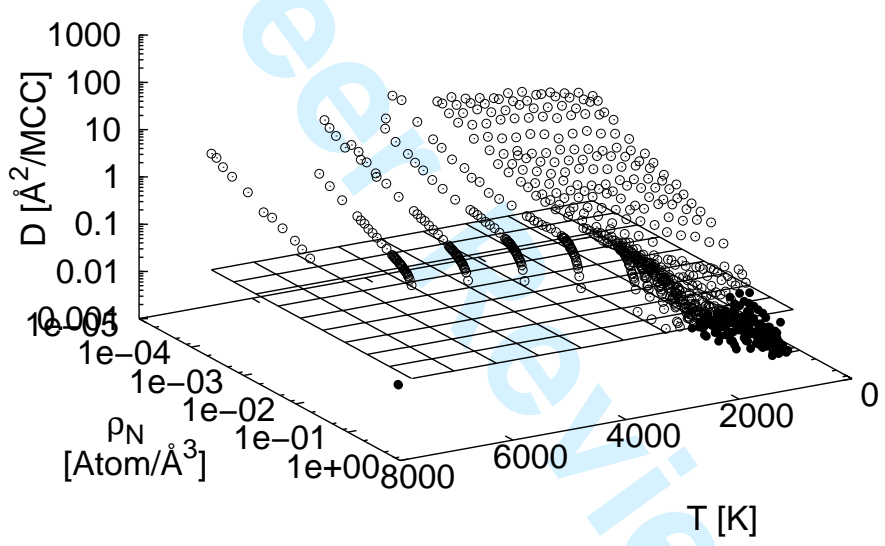

Figure 7: Temperature and density dependence of the diffusion coefficients D. The temperature is given in $K$ and the densities are given in Atoms $/ \AA^{3}$. 


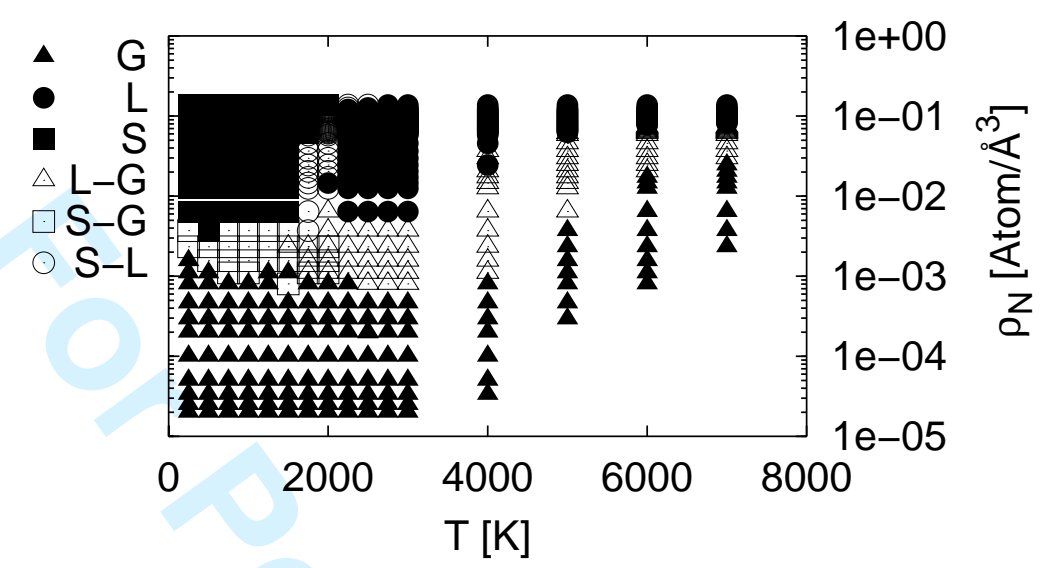

(a) Procedure 1

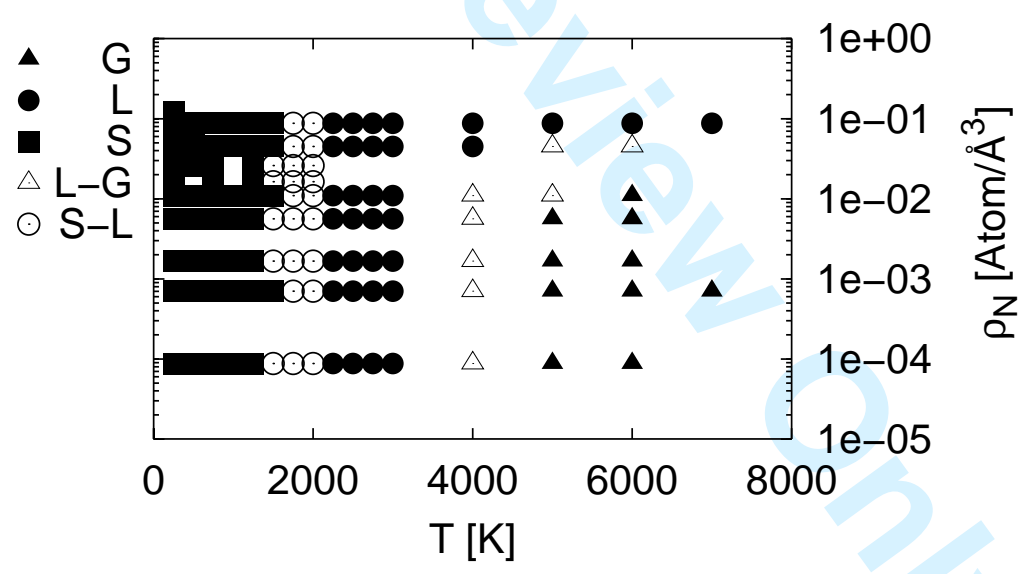

(b) Procedure 2

Figure 8: Estimates of phase diagrams of the $\mathrm{Si}_{3} \mathrm{~B}_{3} \mathrm{~N}_{7}$ system using procedures 1 and 2. G: gas phase, L: liquid phase, S: solid phase, L-G: liquid-gas region, S-L: solid liquid coexistence S-G: solid-gas coexistence. 


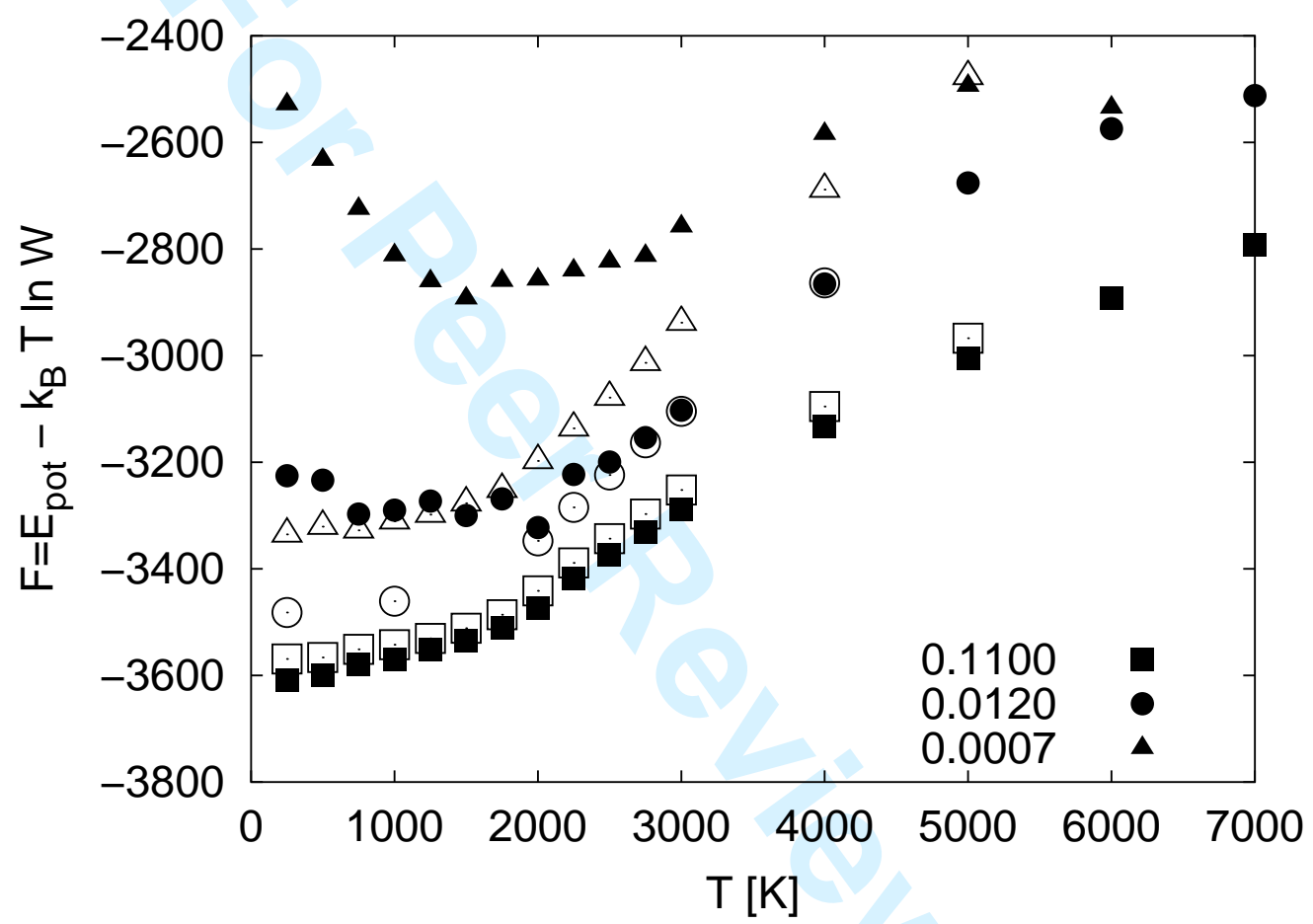

Figure 9: Temperature dependence of the free energy estimates $F^{1,2}(T ; V)$ for procedures 1 (filled symbols) and 2 (open symbols), for three typical isochores. The free energies are given in $\mathrm{eV}$ and the temperature is given in $\mathrm{K}$. Number densities are given inside the figure. 


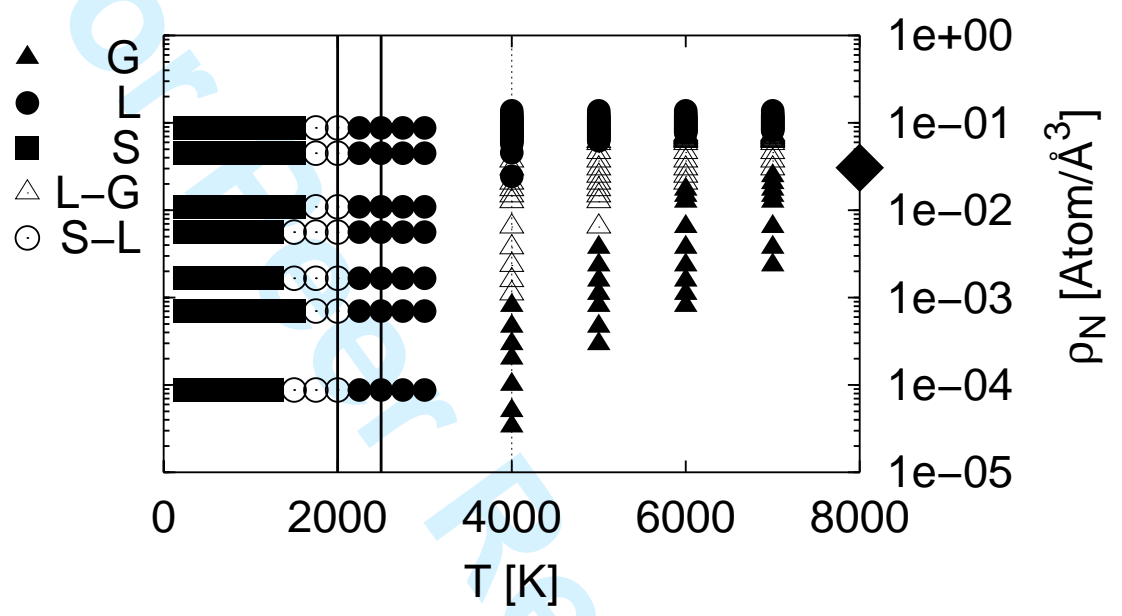

Figure 10: Phase diagram estimate for $\mathrm{Si}_{3} \mathrm{~B}_{3} \mathrm{~N}_{7}$. G: gas phase, L: liquid phase, S: solid phase, L-G: liquid-gas region, S-L: solid liquid coexistence (Here, the crystalline solid is the thermodynamically stable phase compared to the liquid, but the melt can be supercooled, at least on the time scale of the simulations.). The data below $4000 \mathrm{~K}$ (indicated by the dotted vertical line) was taken from procedure 2 and the data above $4000 \mathrm{~K}$ was taken from procedure 1. The large filled diamond is the estimate of the critical point of the $\mathrm{Si}_{3} \mathrm{~B}_{3} \mathrm{~N}_{7}$-system. 


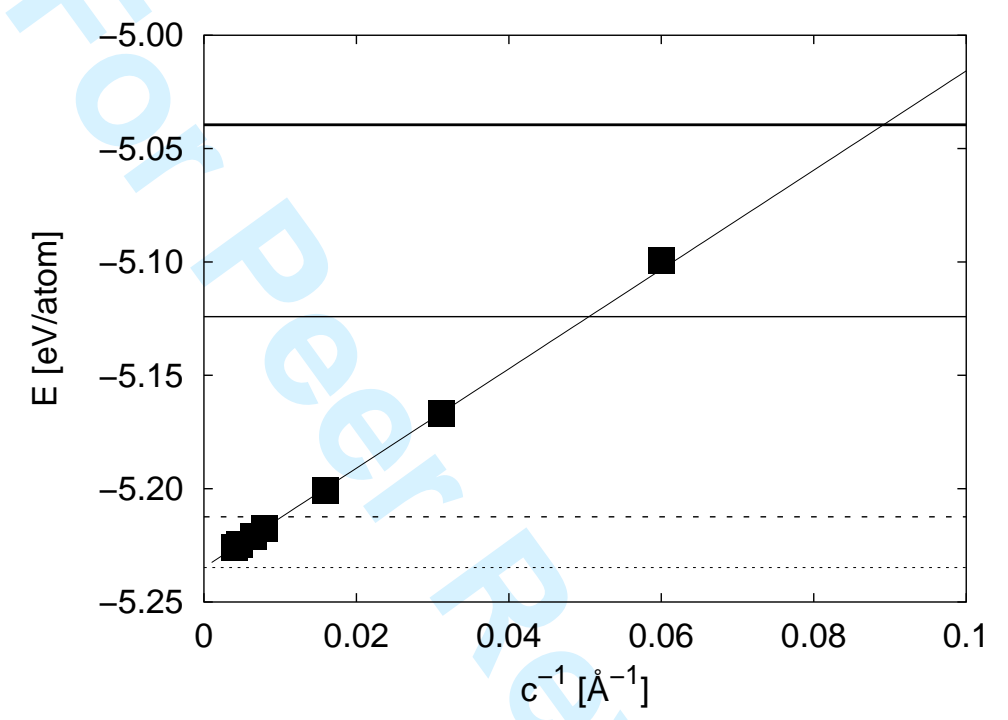

Figure 11: Energy of two-slab configuration as function of inverse cell length $1 / c$ in the z-direction after NpT-relaxation. The fit yields a slope of $m \approx 2.19 \mathrm{eV} /$ atom $\AA$. The dotted line denotes the energy of the phase separated bulk $\beta-\mathrm{Si}_{3} \mathrm{~N}_{4}$ plus h-BN. Note that the extrapolation of the two-slab data reaches this value for infinite thickness of the slabs. In addition, we show the average energies of the metastable ternary crystalline (dashed) and amorphous (solid) solids $\mathrm{Si}_{3} \mathrm{~B}_{3} \mathrm{~N}_{7}$, and the ternary amorphous phase generated by sintering nanocrystallites[14] (bold), respectively. 


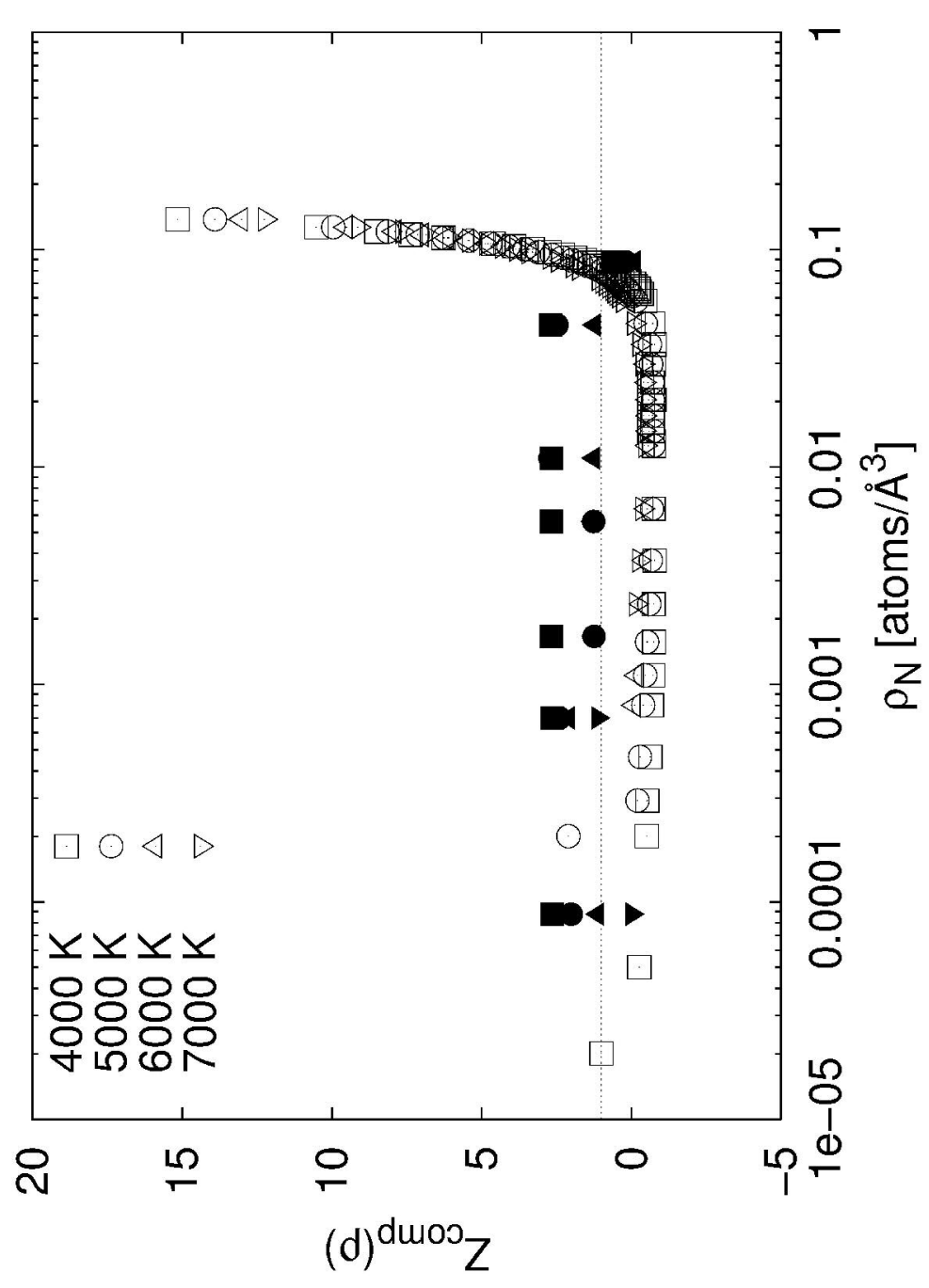

Figure 1a $215 \times 279 \mathrm{~mm}(600 \times 600 \mathrm{DPI})$ 


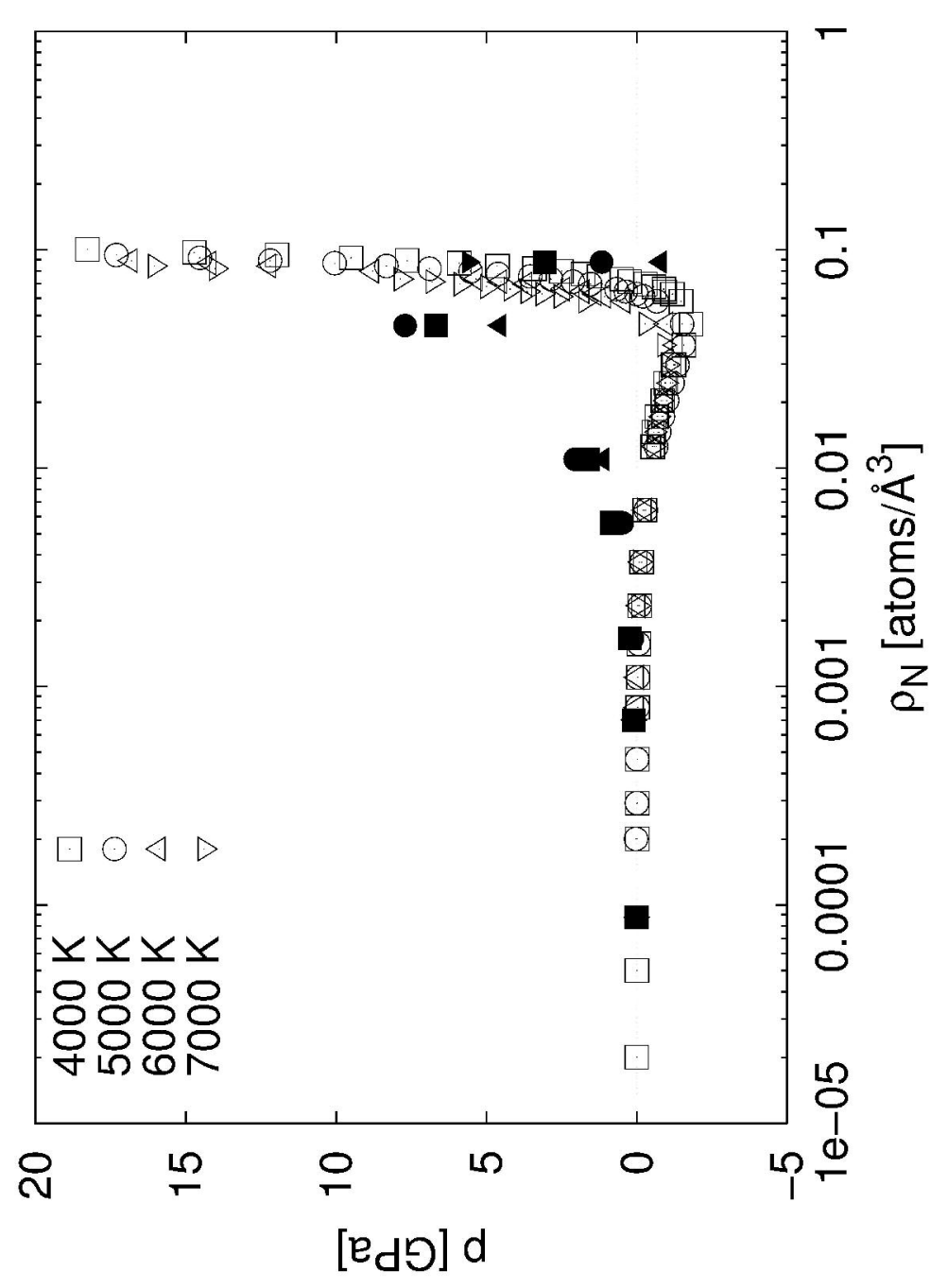

Figure 1b

$215 \times 279 \mathrm{~mm}(600 \times 600 \mathrm{DPI})$

http://mc.manuscriptcentral.com/pm-pml 


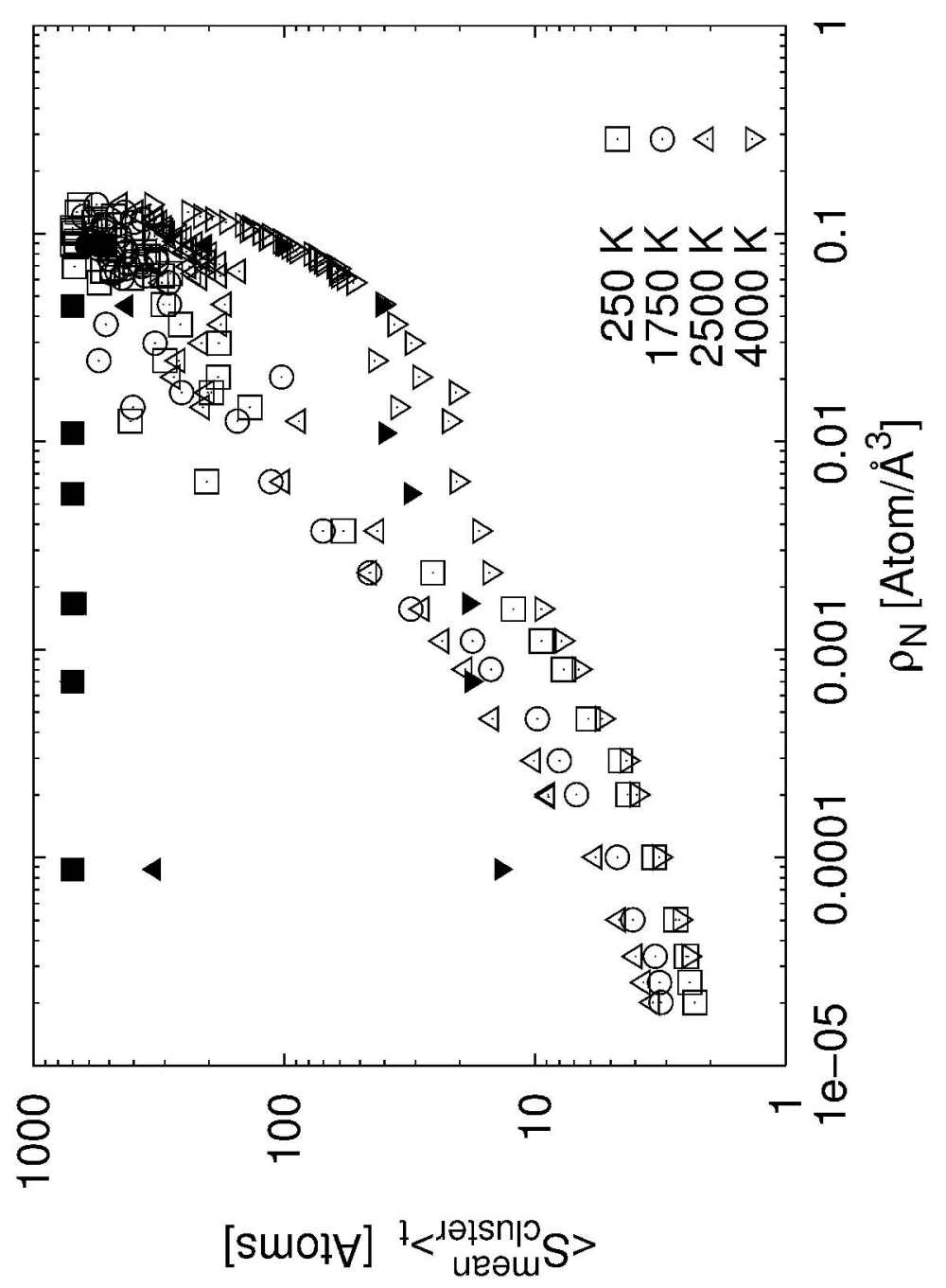

Figure 2a

$215 \times 279 \mathrm{~mm}(600 \times 600 \mathrm{DPI})$ 


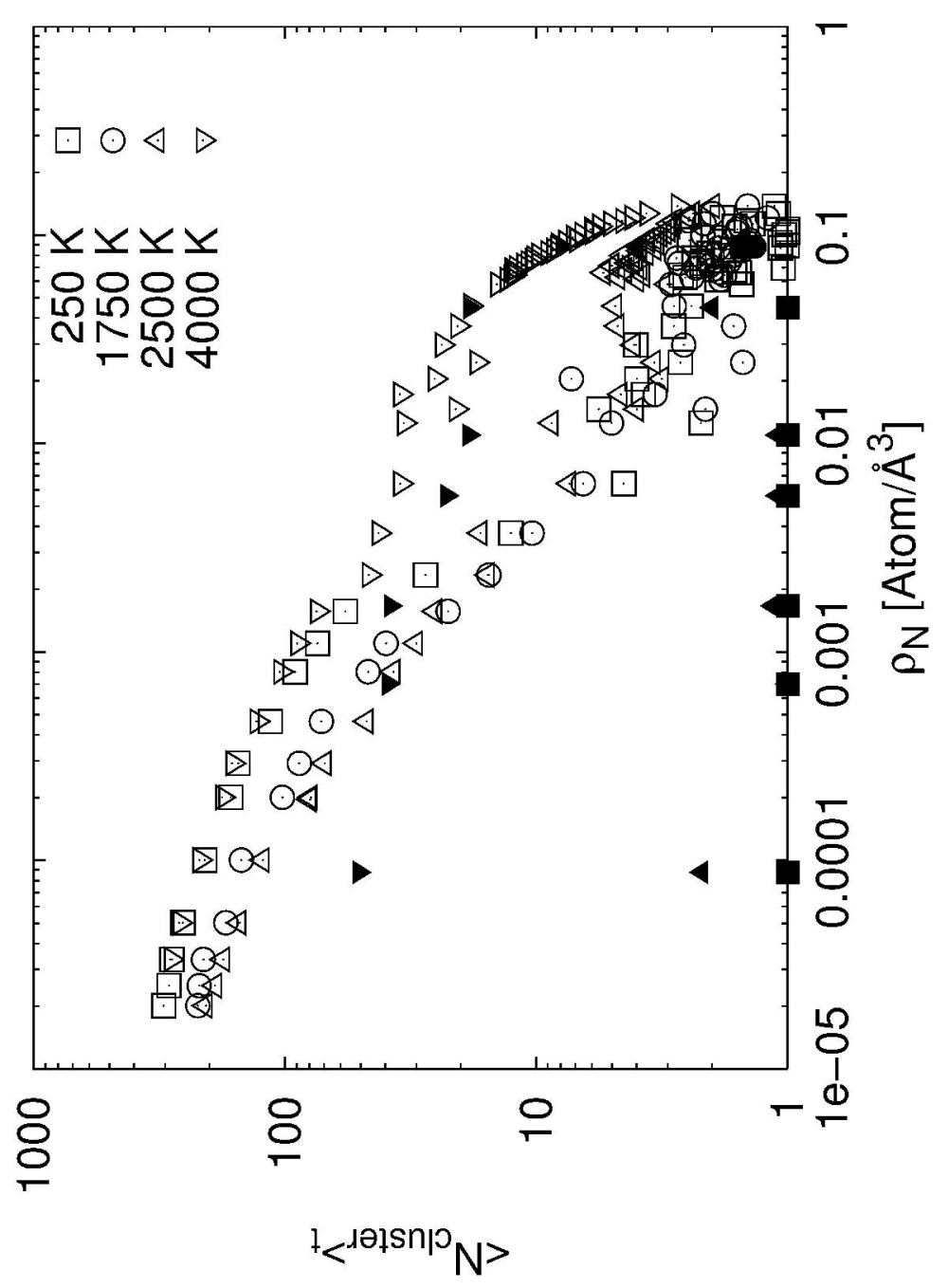

Figure 2b

$215 \times 279 \mathrm{~mm}(600 \times 600 \mathrm{DPI})$ 


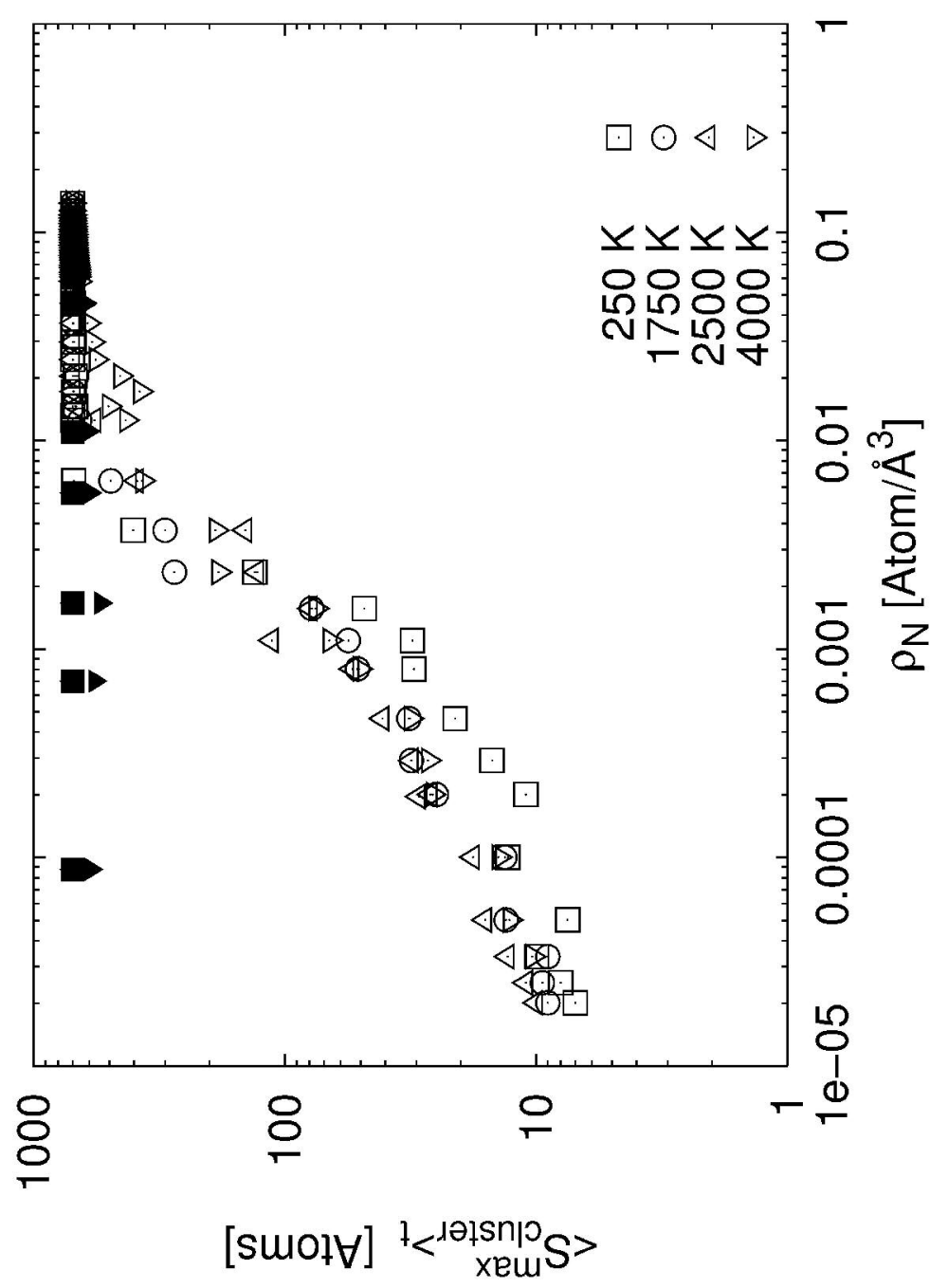

Figure 2c

$215 \times 279 \mathrm{~mm}(600 \times 600 \mathrm{DPI})$ 


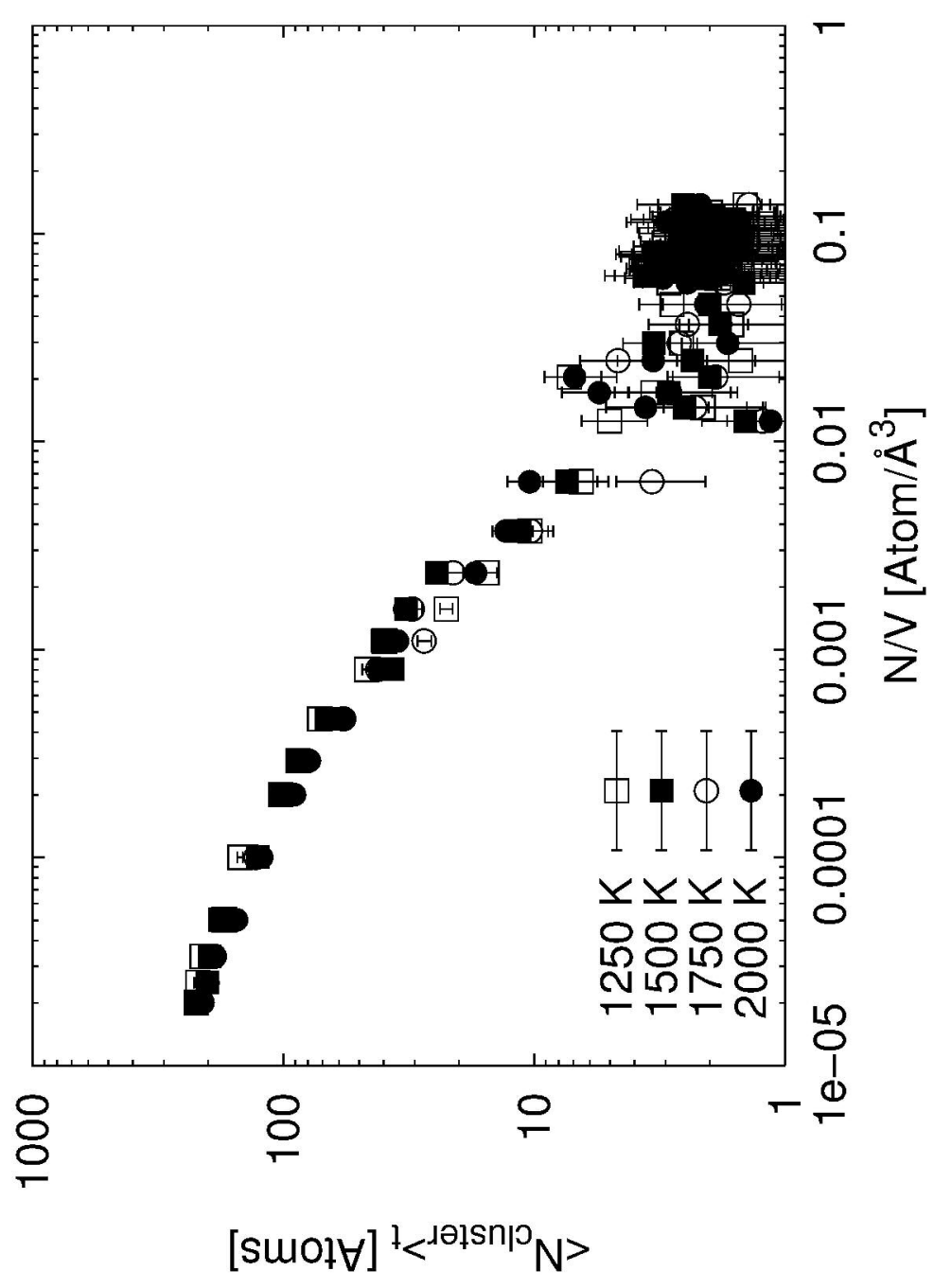

Figure 3a (left)

$215 \times 279 \mathrm{~mm}(600 \times 600 \mathrm{DPI})$ 


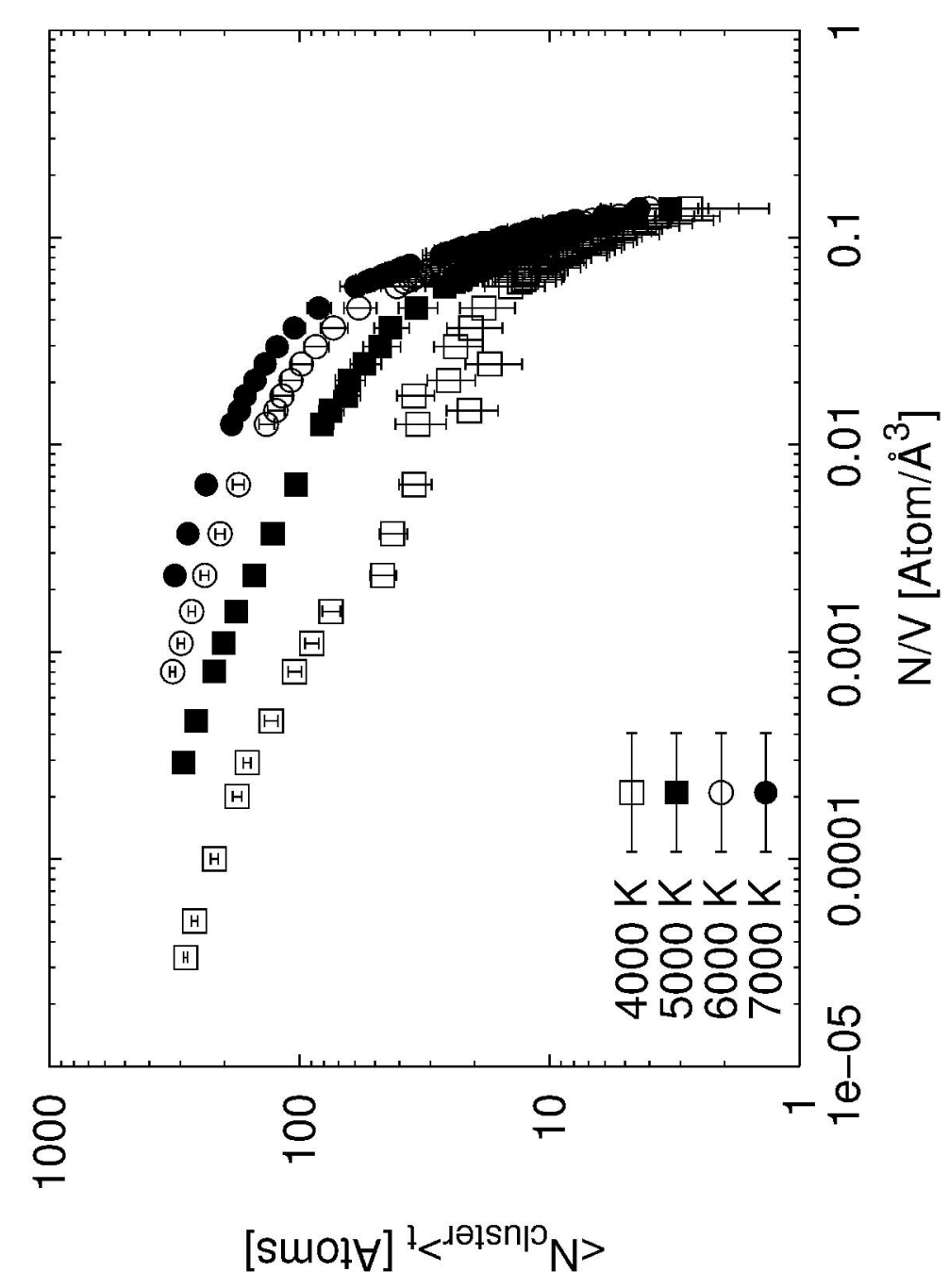

Figure 3a (right)

$215 \times 279 \mathrm{~mm}(600 \times 600 \mathrm{DPI})$ 


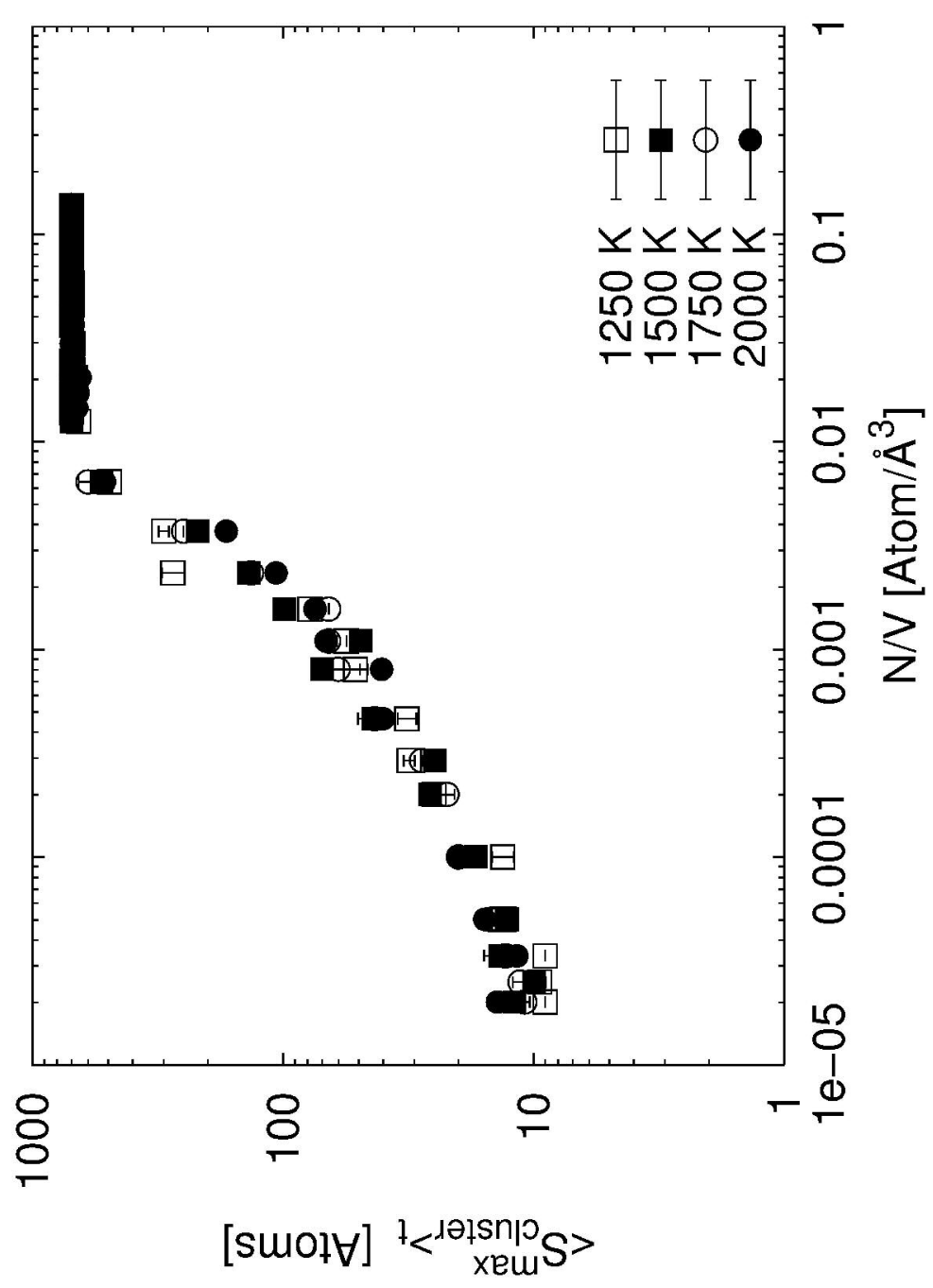

Figure 3b (left)

$215 \times 279 \mathrm{~mm}(600 \times 600 \mathrm{DPI})$ 


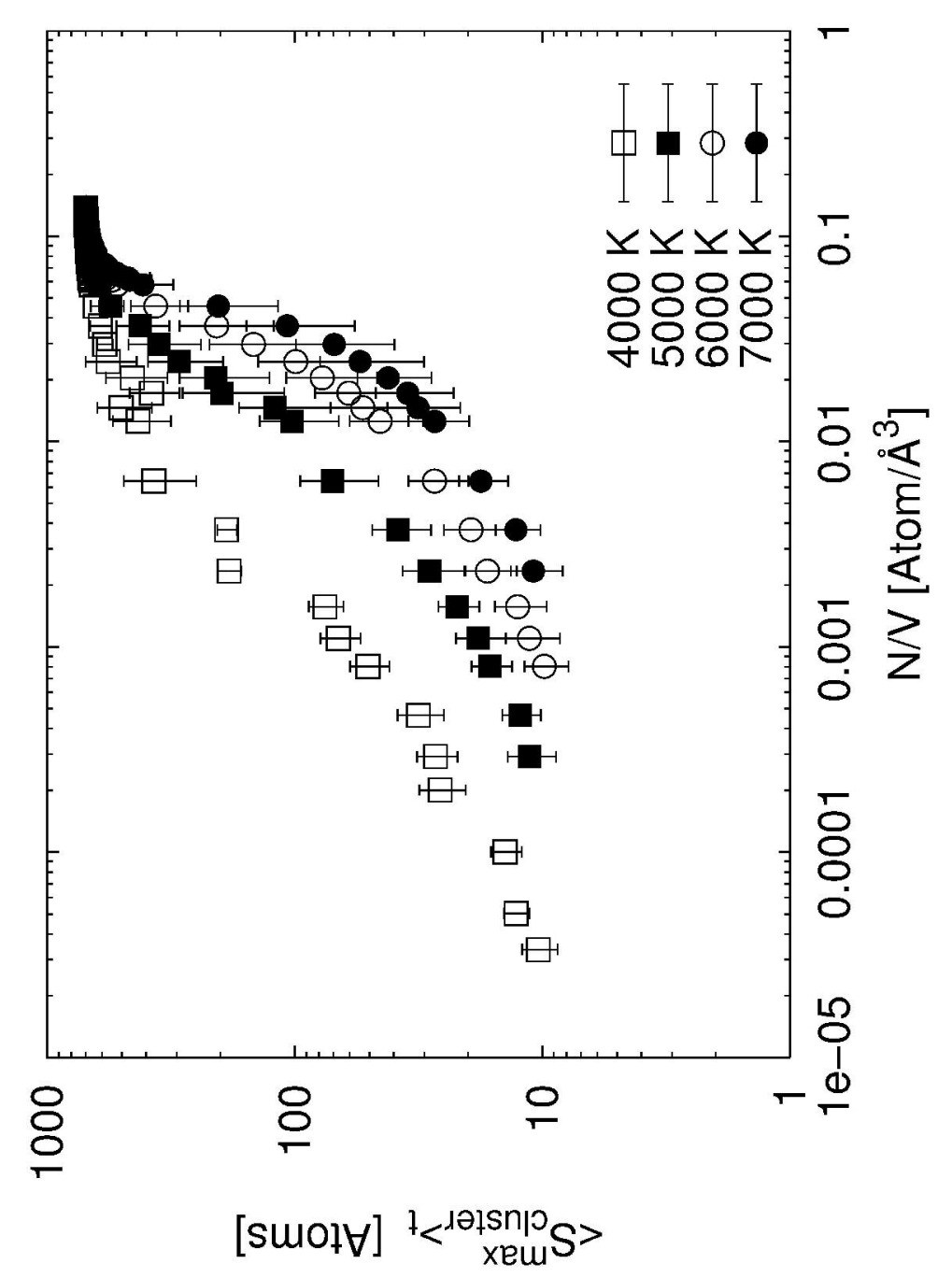

Figure 3b (right)

$215 \times 279 \mathrm{~mm}(600 \times 600$ DPI $)$ 

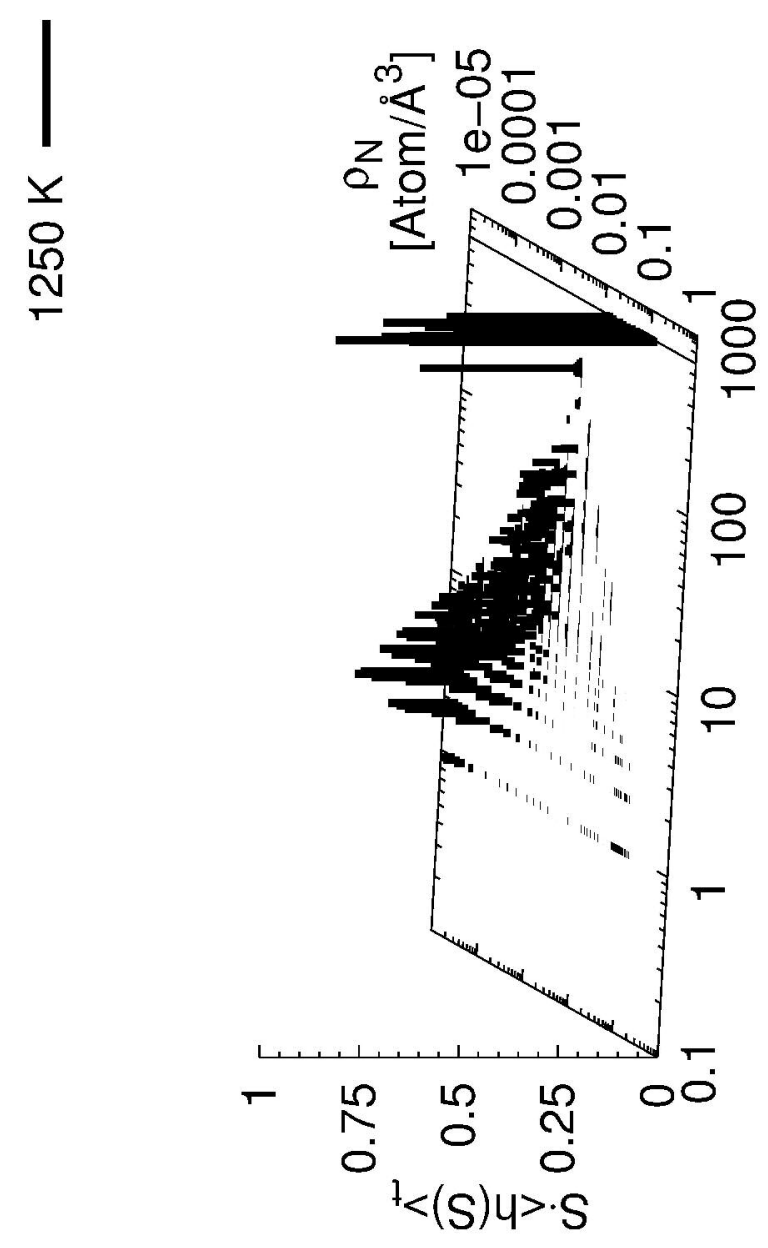

Figure 4a (top) $215 \times 279 \mathrm{~mm}(600 \times 600 \mathrm{DPI})$ 

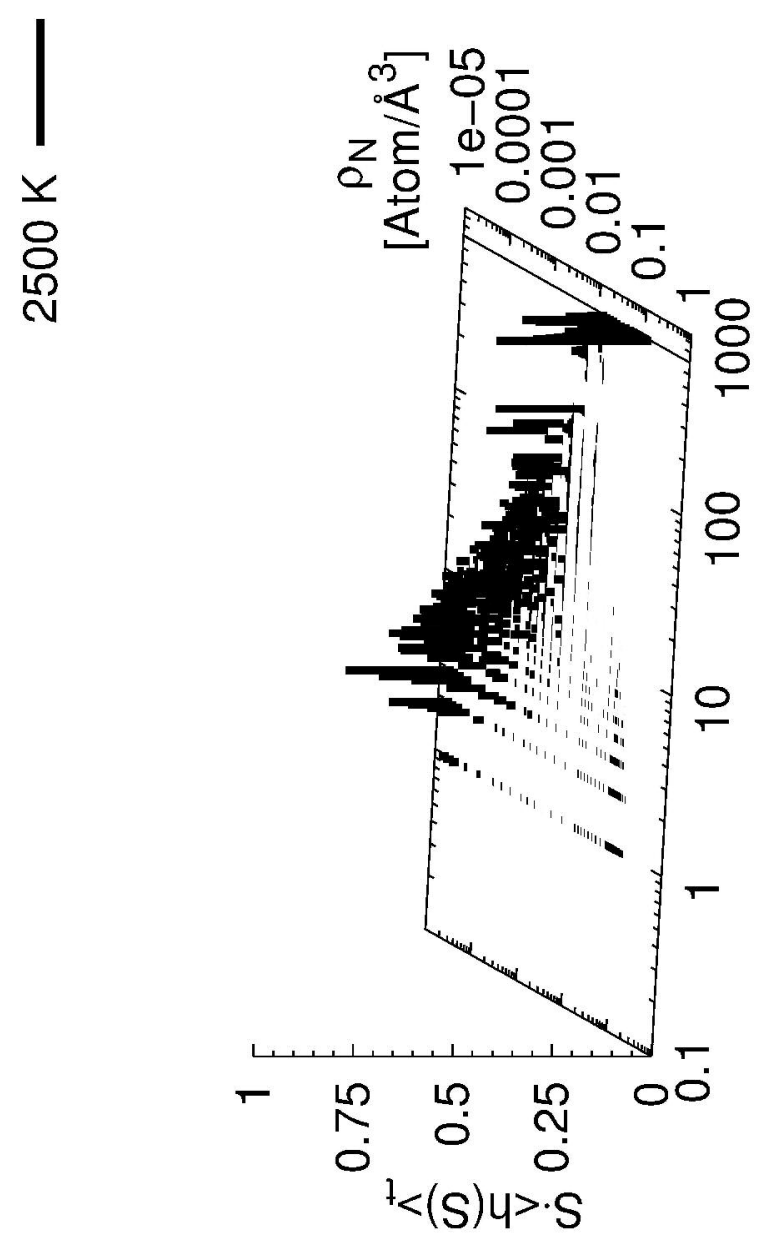

Figure 4a (middle) $215 \times 279 \mathrm{~mm}(600 \times 600 \mathrm{DPI})$ 

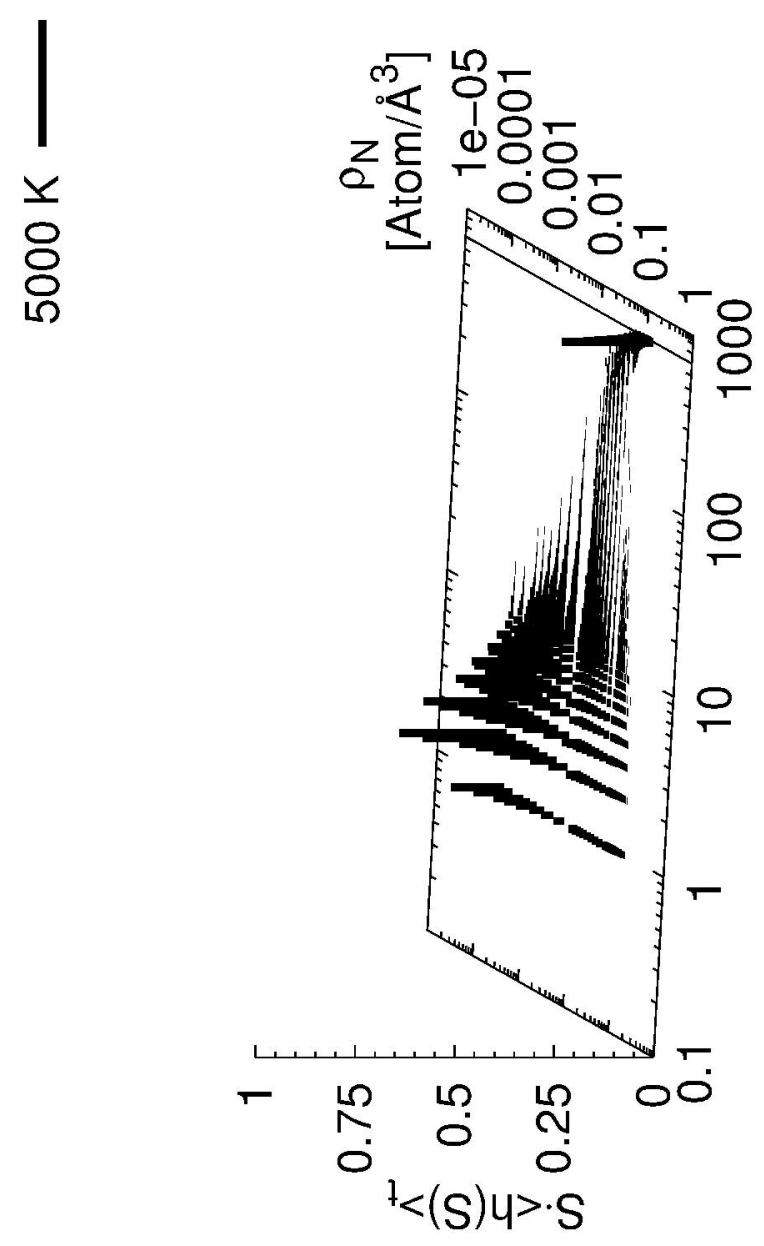

Figure 4a (bottom) $215 \times 279 \mathrm{~mm}(600 \times 600 \mathrm{DPI})$ 


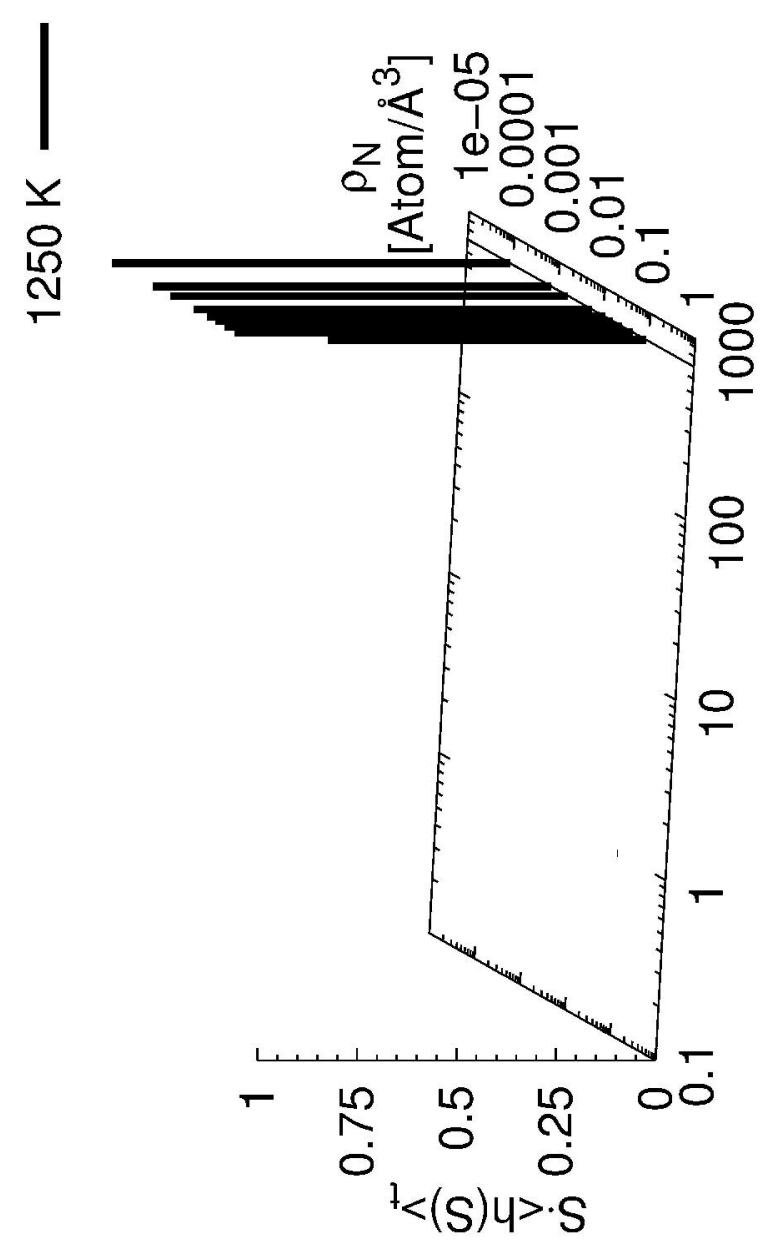

Figure 4b (top) $215 \times 279 \mathrm{~mm}(600 \times 600 \mathrm{DPI})$ 


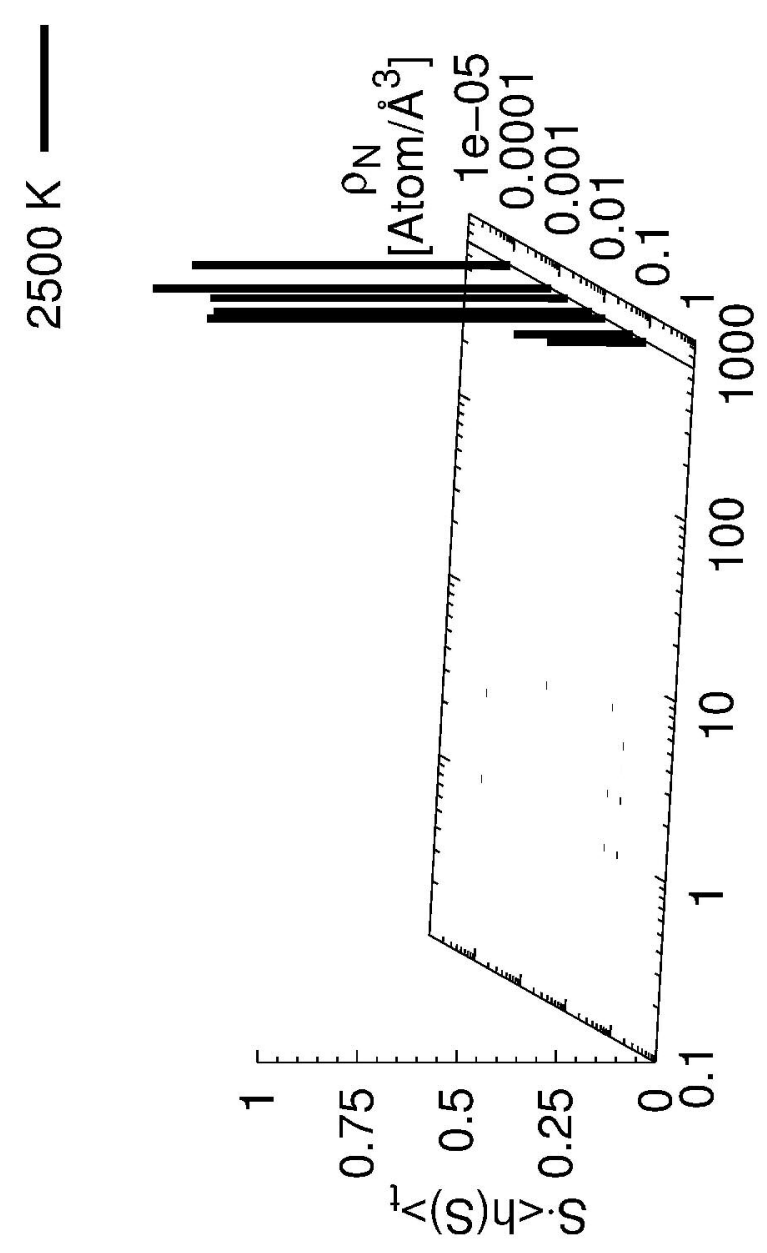

Figure 4b (middle) $215 \times 279 \mathrm{~mm}(600 \times 600 \mathrm{DPI})$ 

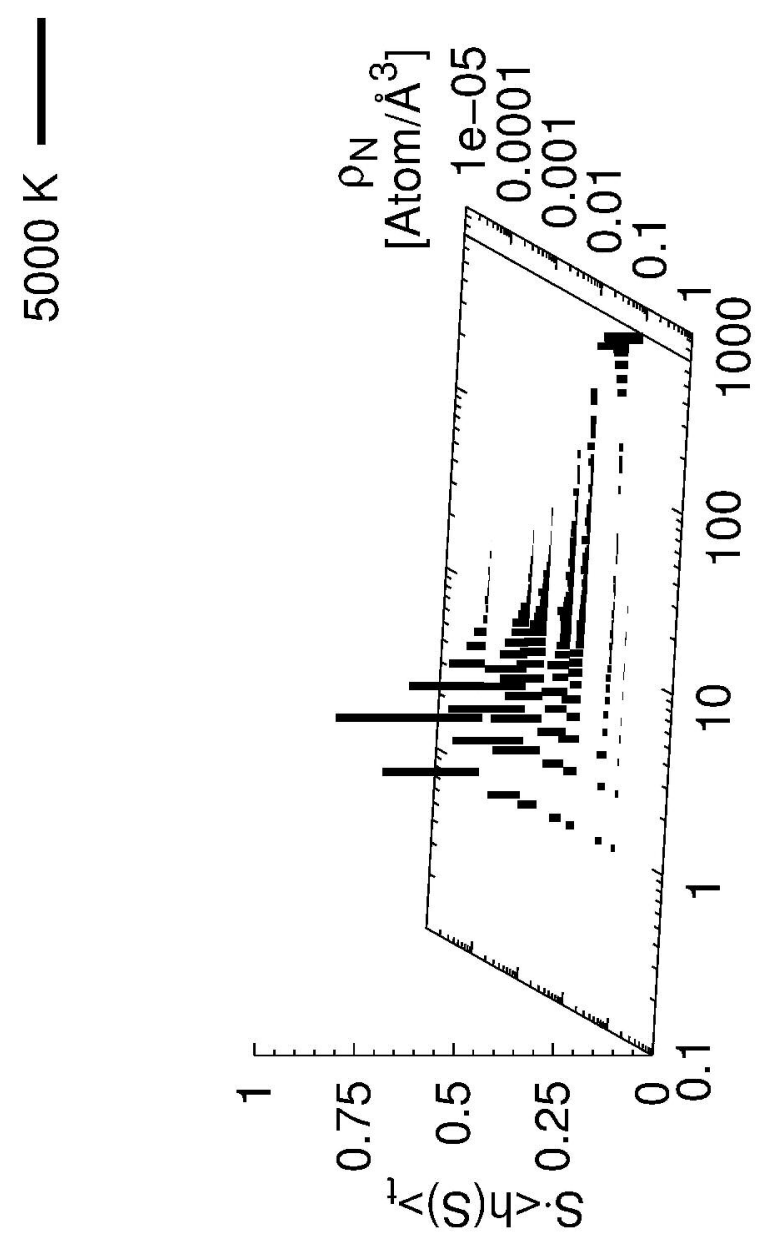

Figure 4b (bottom) $215 \times 279 m m(600 \times 600$ DPI $)$ 


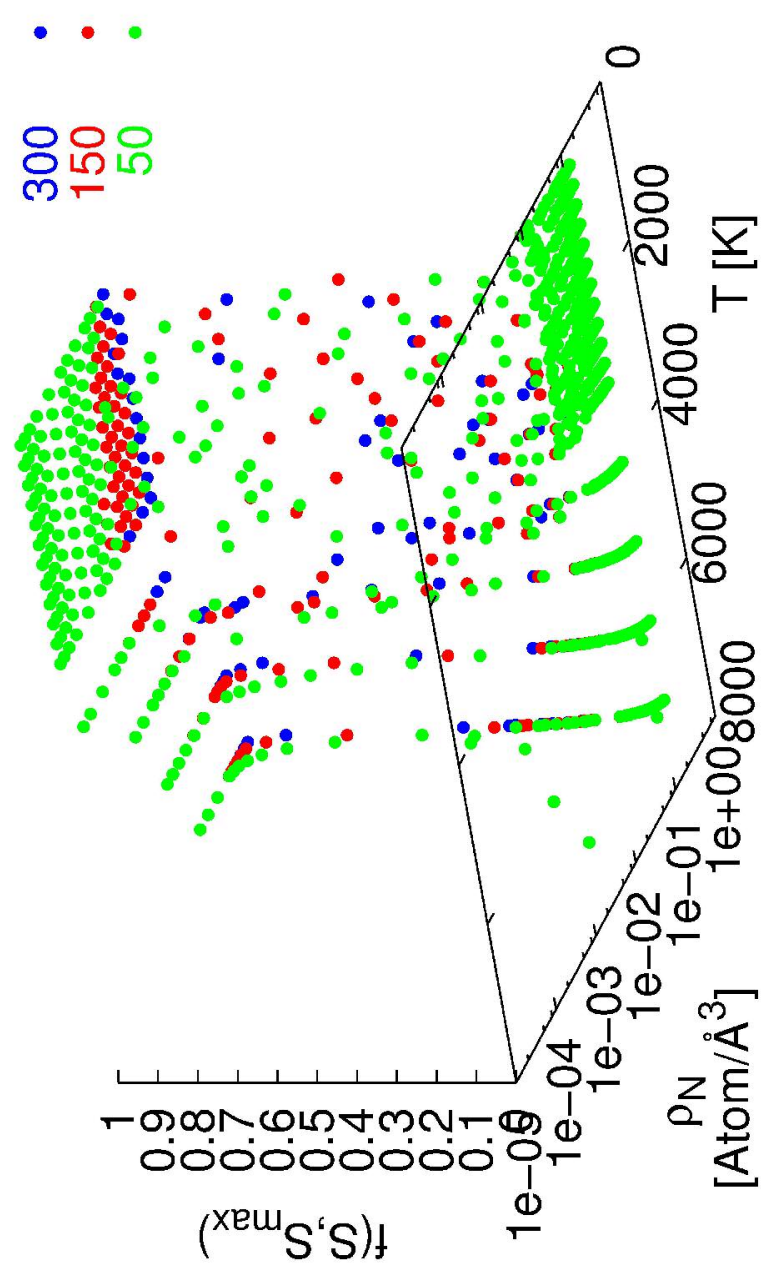

Figure 5

$215 \times 279 \mathrm{~mm}(600 \times 600 \mathrm{DPI})$ 


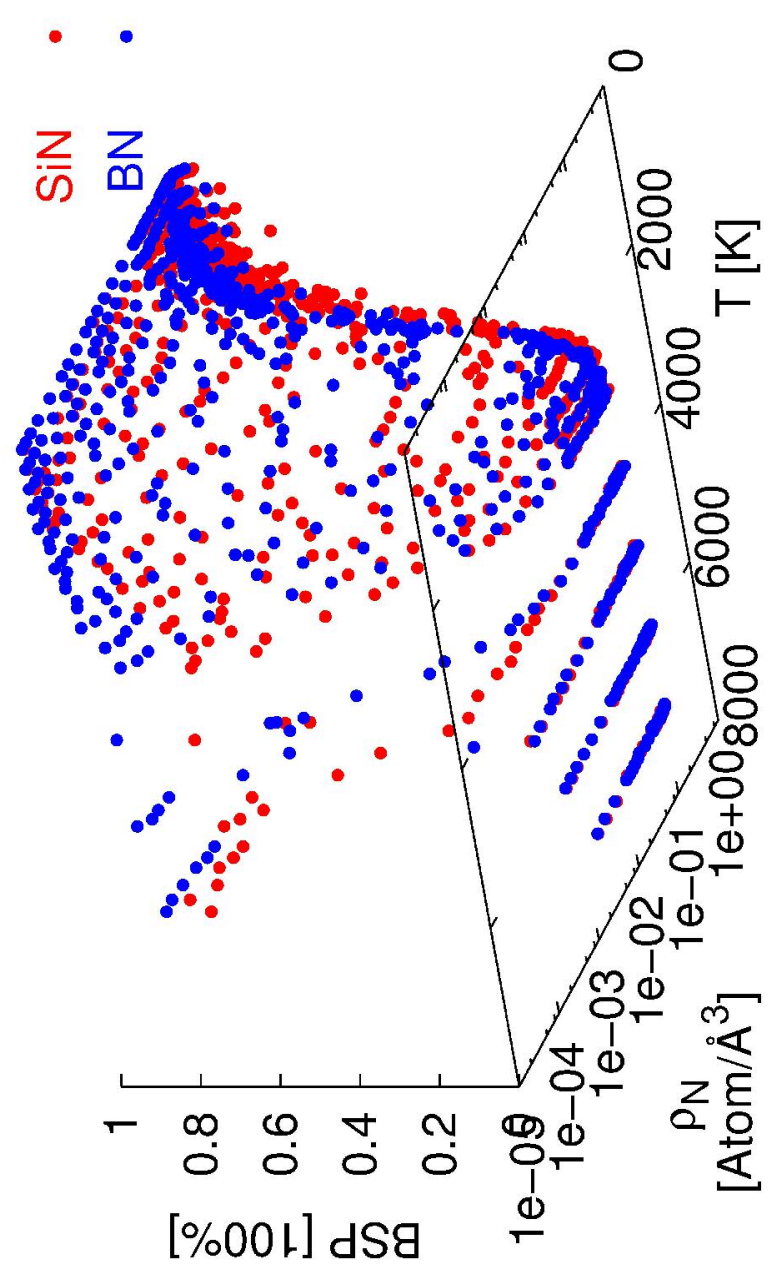

Figure 6a

$215 \times 279 \mathrm{~mm}(600 \times 600 \mathrm{DPI})$ 


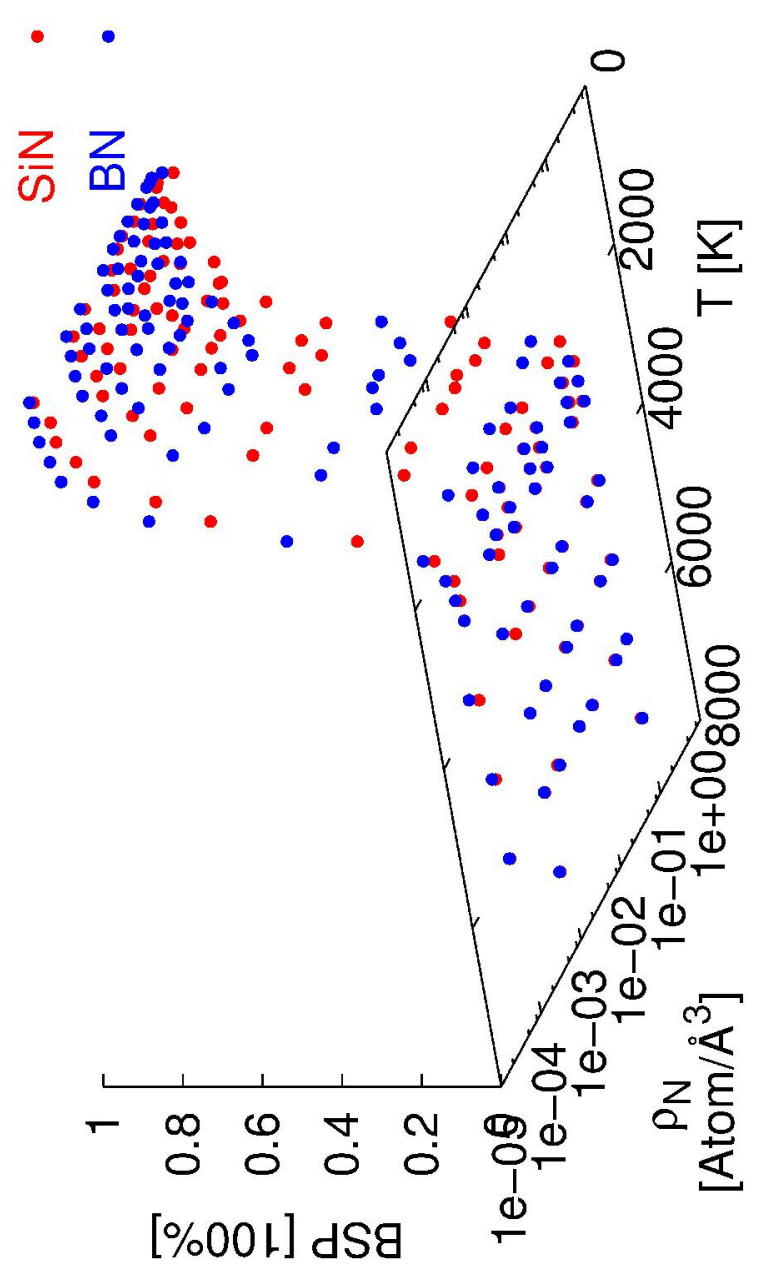

Figure 6b

$215 \times 279 \mathrm{~mm}(600 \times 600 \mathrm{DPI})$ 


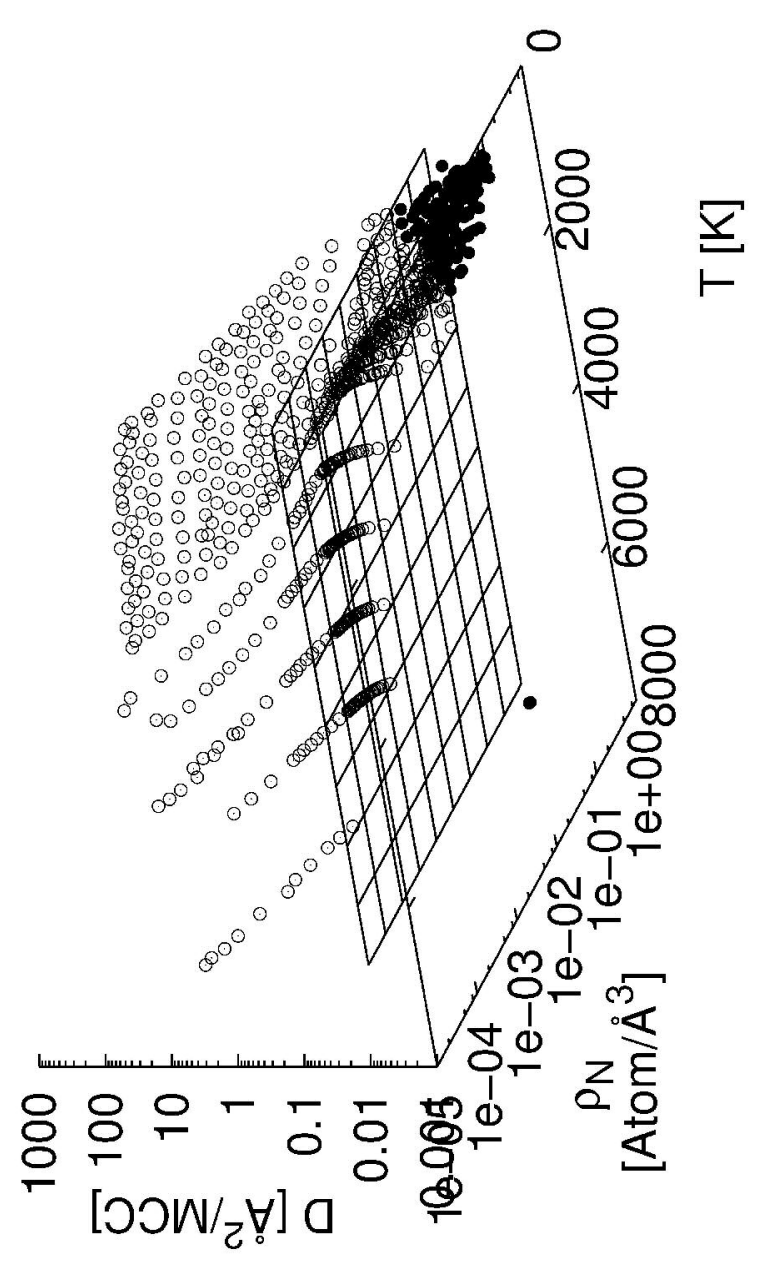

Figure 7

$215 \times 279 \mathrm{~mm}(600 \times 600 \mathrm{DPI})$ 


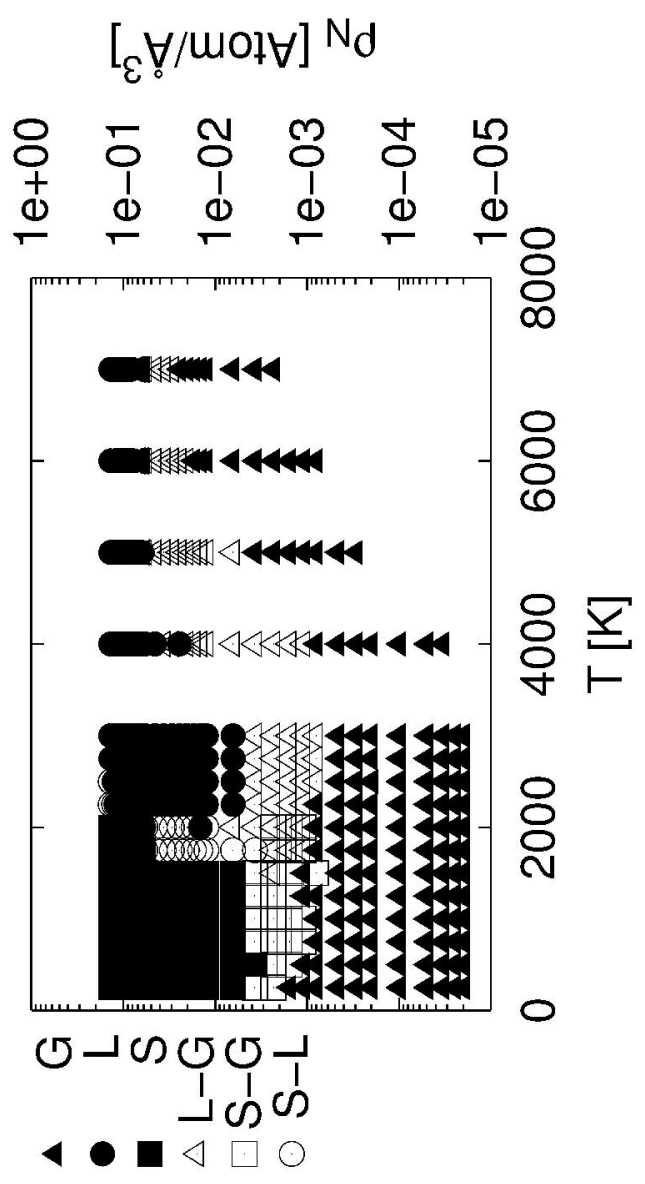

Figure 8a

$215 \times 279 \mathrm{~mm}(600 \times 600 \mathrm{DPI})$ 


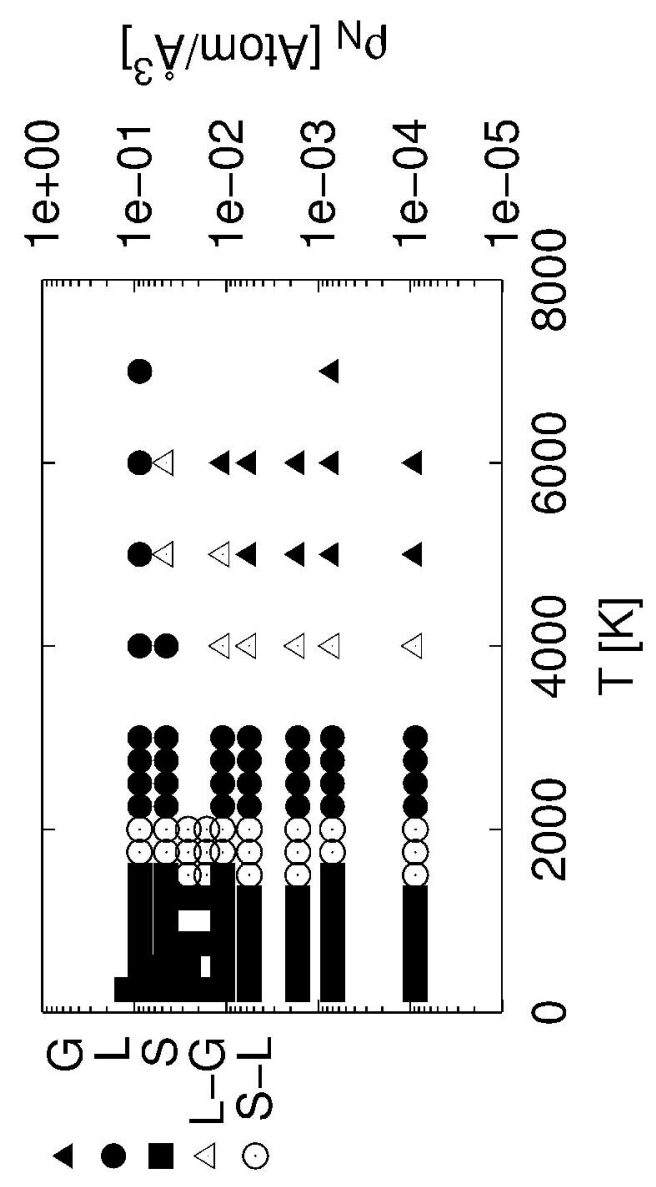

Figure 8b

$215 \times 279 \mathrm{~mm}(600 \times 600$ DPI $)$ 


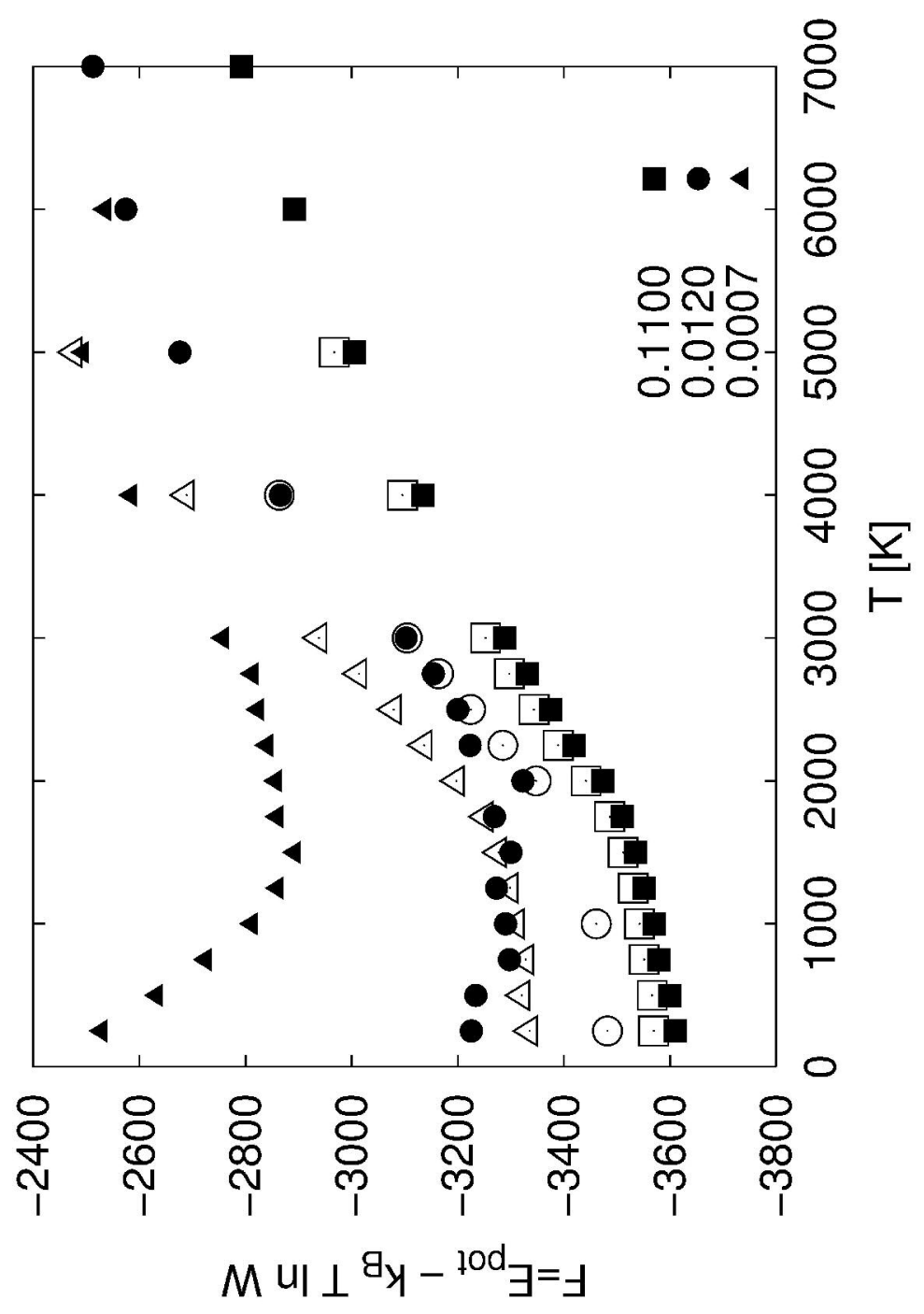

Figure 9

$215 \times 279 \mathrm{~mm}(600 \times 600 \mathrm{DPI})$ 


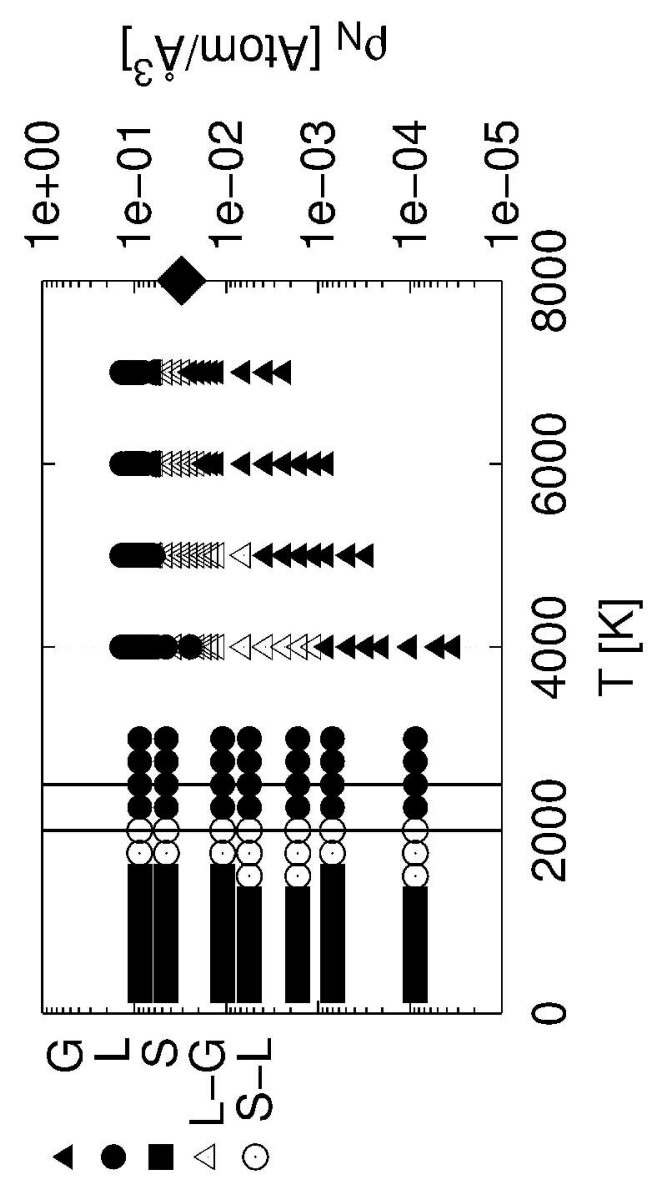

Figure 10

$215 \times 279 \mathrm{~mm}(600 \times 600 \mathrm{DPI})$ 


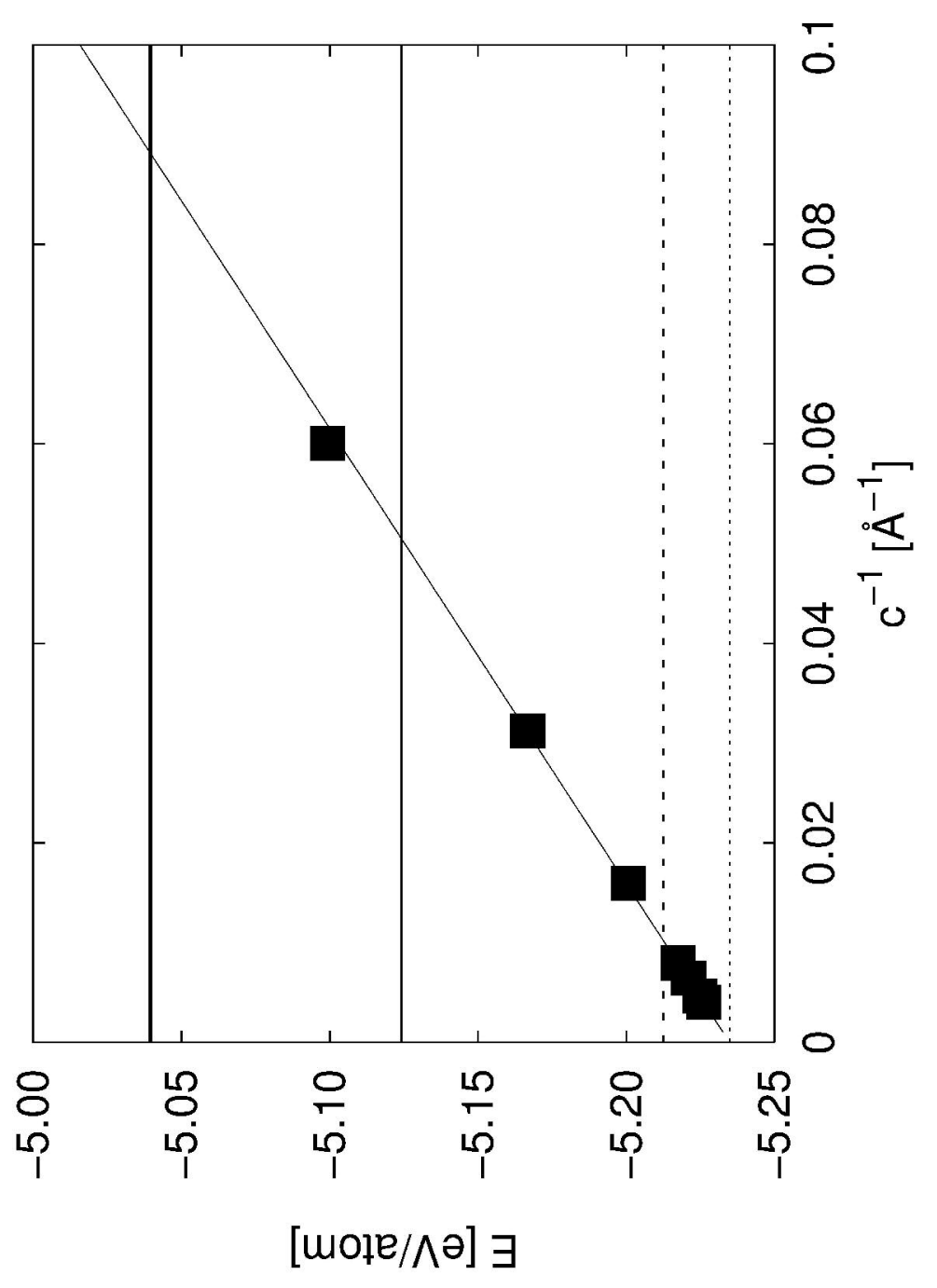

Figure 11

$215 \times 279 \mathrm{~mm}(600 \times 600 \mathrm{DPI})$ 